\title{
FULLY MASS-CONSERVATIVE IMPES SCHEMES FOR INCOMPRESSIBLE TWO-PHASE FLOW IN POROUS MEDIA
}

\author{
HUANGXIN CHEN, JISHENG KOU, SHUYU SUN*, AND TAO ZHANG
}

\begin{abstract}
In this paper we consider fully mass-conservative numerical schemes for the simulation of incompressible and immiscible two-phase flow in porous media with capillary pressure. Compared with two kinds of conventional IMplicit Pressure Explicit Saturation (IMPES) schemes, the new schemes deserve a merit that the conservation of mass of both phases can be obtained. The total conservation equation is obtained by the summation of the discretized conservation equation for each phase. This approach is quite different from the conventional IMPES schemes. We present two kinds of fully mass-conservative IMPES schemes to solve the coupled systems for pressure, auxiliary velocity and saturation of each phase. The upwind mixed finite element methods are used to solve the pressure-velocity systems which can be decoupled, and the problems in the decoupled systems can be proved to be well-posed. Moreover, the new schemes are unbiased and the saturation of each phase can be proved to be bounds-preserving if the time step size is smaller than a certain value. The new schemes can also be applied to approximate the incompressible and immiscible two-phase flow in heterogeneous porous media with different capillarity pressures. Several interesting examples of incompressible and immiscible two-phase flow in porous media are presented to demonstrate the robustness of the new algorithms.
\end{abstract}

\section{INTRODUCTION}

Modeling and simulation of two-phase flow in porous media are of interest in hydrology and petroleum reservoir engineering. Mathematical model of two-phase flow in porous media is a coupled system of timedependent nonlinear PDEs which include the Darcy's law, the equation of conservation of mass for each phase, the constraint of the saturations of phases, and the equation of capillary pressure which depends on the wetting saturation. The fully implicit scheme [5, 17, 19, 43, 48, 49, 50, 51, 54] implicitly treats all the terms and solves all the unknowns simultaneously, thus it deserves unconditional stability. However, the implicit methods require lots of computational resources per time step. The IMplicit-EXplicit (IMEX) scheme $[4,8,25,30,36]$ treats the linear terms implicitly and evaluates the others explicitly, which deserves better stability than the fully explicit scheme and may eliminate the nonlinearity of the original equations.

Operator splitting $[3,24,38]$ is an efficient approach to reduce a complex time-dependent problem into several simpler problems. By this approach, one can further construct iterative operator-splitting schemes $[24,26,31]$ which can be viewed as an iterative approach to solve the nonlinear system. The IMplicit Pressure Explicit Saturation (IMPES) scheme [11, 12, 14, 15, 16, 23, 45, 46, 52] has been widely used to solve the coupled nonlinear system for the two-phase flow in porous media. It can be viewed as an IMEX scheme employing a splitting approach based on physics. In the standard IMPES scheme [45, 46], the pressure equation is obtained by substituting the saturation constraint and Darcy's law in the summation of the two mass conservation laws for each phase at time and space continuous level, and all other variables in the pressure equation are explicitly treated to eliminate the nonlinearity. Then the pressure equation is solved implicitly, and the Darcy's velocity and the saturations of both phases can be updated explicitly as long as the pressure is obtained. This method is simple to set up and efficient to implement, and requires less computer memory at each time step compared with the fully implicit scheme.

2010 Mathematics Subject Classification. 65M60,65N30,76S05.

Key words and phrases. Two-phase flow, IMPES, upwind mixed finite element method, conservation of mass, boundspreserving, unbiased.

*Corresponding author: Shuyu Sun (shuyu.sun@kaust.edu.sa).

The work of Huangxin Chen was supported by the NSF of China (Grants No. 11771363, 91630204, 51661135011), the Fundamental Research Funds for the Central Universities (Grant No. 20720180003) and Program for Prominent Young Talents in Fujian Province University. The work of Tao Zhang and Shuyu Sun was supported by funding from King Abdullah University of Science and Technology (KAUST) through the grants BAS/1/1351-01, URF/1/2993-01, and REP/1/2879-01. 
For different capillary pressure functions in case of heterogeneous porous media, the standard IMPES scheme may not be suitable because of its inconsistency. A revised IMPES formulation was proposed by Hoteit and Firoozabadi [27, 28] (HF-IMPES) to treat contrast in capillary pressure of heterogeneous porous media, which can have a significant effect on the flow path in two-phase immiscible flow. The HF-IMPES scheme is based on the conception that the wetting phase pressure is always continuous as long as none of the phases is immobile, and can reproduce the saturation solution with expected discontinuity. Both the standard IMPES and HF-IMPES schemes are mass-conservative for the wetting phase and produce nonnegative wetting-phase saturation if the time step size is smaller than a certain value. However, both the two IMPES schemes are only mass-conservative for the wetting phase, and thus are not mass-conservative for the total fluid mixture. Moreover, both the two IMPES schemes might produce a wetting-phase saturation that larger than one.

Various improved IMPES schemes have been proposed for the simulation of two-phase flow in porous media (cf. e.g. $[1,11,12,20,21,29,32,33,34,35,42,44,22,47,52]$ and the references therein). The iterative IMPES scheme is to use IMPES as an iterative step of the fully implicit scheme instead of Newton iteration. Another iterative scheme developed in [37, 40, 41] solves an implicit pressure equation and an implicit saturation equation in each iteration, and the implicit saturation equation is derived from the implicit capillary pressure. An efficient iterative IMPES scheme [33] was further proposed based on the HFIMPES scheme for the two-phase immiscible flow in porous media with different capillarity pressures. The computational cost and memory required by the iterative IMPES scheme is smaller than the iterative method for the fully implicit scheme, thus it is more feasible for the problems with large system. Recently, Kou and Sun [35] proposed an improved IMPES scheme for the incompressible and immiscible two-phase flow in porous media. Different from the standard IMPES scheme and the HF-IMPES scheme, the pressure equation in [35] was obtained by summing the discretized conservation equations of two phases. This treatment yields a merit that the conservation of mass holds for both phases instead of only one phase in the conventional IMPES methods. In [35], the wetting and non-wetting phases pressures are both unknowns in the pressure system, and the interior penalty discontinuous Galerkin with upwind scheme is used in the discrete formulation.

Since the algorithms for the standard IMPES and HF-IMPES schemes are biased, and the two schemes are mass-conservative for only one phase and yield saturations which may be larger than one, this motivates us to develop fully mass-conservative IMPES schemes for the simulation of incompressible and immiscible two-phase flow in porous media in this work. Two kinds of fully mass-conservative IMPES schemes will be presented to solve the coupled system for pressure, auxiliary velocity and saturation for both phases. The total conservation equation is obtained by summing the discretized conservation equation for each phase, and the mixed finite element method with upwind scheme is used in the pressure-velocity system. Then combining the constraint of the saturations of phases, the equation of capillary pressure and the total conservation equation, we can obtain the coupled nonlinear system for pressure and auxiliary velocity of both phases. In [35], the authors obtained the pressure system which couples both pressures in the associated IMPES scheme, and the computational cost will be more expensive than the conventional IMPES schemes. Different from the coupled pressure system in [35], when the wetting saturation is given, the coupled pressure-velocity system in this work can be decoupled into two decoupled systems which can be proved to be well-posed, and the wetting and non-wetting pressure and auxiliary velocity can be implicitly solved. Then the saturations of both phases can be explicitly obtained. Moreover, the new two kinds of fully mass-conservative IMPES schemes are unbiased and the saturation of each phase is bounds-preserving if the time step size is smaller than a certain value. The discrete total velocity can be recovered by post-processing to be continuous in the normal direction in the whole domain. Moreover, we remark that the newly designed fully mass-conservative IMPES schemes can also be used to approximate the incompressible and immiscible two-phase flow in heterogeneous porous media with different capillarity pressures.

For the fully mass-conservative IMPES schemes of the first kind, we use auxiliary velocity unknowns which should be multiplied by phase mobility to be phase velocity in the pressure-velocity system, and we assume the auxiliary velocity unknowns are continuous in the normal direction in the whole domain. In order to make the schemes to be more physical in some sense, for the fully mass-conservative IMPES schemes of the second kind, we use another kind of auxiliary velocity unknowns in the pressure-velocity system, which will become total velocity when the capillary pressure and gravity effects are disregarded, and it is more physical to assume that the total velocity is continuous in the normal direction in the whole domain. 
The rest of the paper is organized as follows. In Section 2, we introduce the mathematical model for the incompressible and immiscible two-phase flow in porous media and the two conventional IMPES schemes, i.e., the standard IMPES scheme and the HF-IMPES scheme. In Section 3, we propose two kinds of fully mass-conservative IMPES schemes, and some merits of the new schemes will be presented and proved in Section 4. In Section 5, we give some numerical results to verify the robustness of the proposed new schemes. Finally we provide a conclusion in Section 6 .

\section{Mathematical model and COnVEntional IMPES SChemes}

In the following model, the wetting phase and non-wetting phase are denoted by the subscripts $w$ and $n$ respectively. The formulations of incompressible and immiscible two-phase flow in porous media $\Omega$ with gravity are given by

$$
\begin{array}{ll}
\phi \frac{\partial S_{\alpha}}{\partial t}+\nabla \cdot \boldsymbol{u}_{\alpha}=F_{\alpha}, & \text { in } \Omega, \quad \alpha=w, n, \\
\boldsymbol{u}_{\alpha}=-\frac{k_{r \alpha}}{\mu_{\alpha}} \boldsymbol{K}\left(\nabla p_{\alpha}+\rho_{\alpha} g \nabla z\right), & \text { in } \Omega, \quad \alpha=w, n, \\
S_{n}+S_{w}=1, & \text { in } \Omega, \\
p_{c}\left(S_{w}\right)=p_{n}-p_{w}, & \text { in } \Omega,
\end{array}
$$

where $\phi$ is the porosity of the medium, $\boldsymbol{K}$ denotes the absolute permeability tensor, $S_{\alpha}, \boldsymbol{u}_{\alpha}, p_{\alpha}, F_{\alpha}$ are the saturation, Darcy's velocity, pressure and the sink/source term of each phase $\alpha, p_{c}$ is the capillary pressure. In (2.2), $\rho_{\alpha}, k_{r \alpha}, \mu_{\alpha}$ are the density, relative permeability and viscosity of phase $\alpha, g$ is the magnitude of the gravitational acceleration, $z$ is the depth. The phase mobility is defined by $\lambda_{\alpha}=\frac{k_{r_{\alpha}}}{\mu_{\alpha}}$, and the total mobility is given by $\lambda_{t}=\lambda_{w}+\lambda_{n}$. We also define the fractional flow functions as

$$
f_{w}=\lambda_{w} / \lambda_{t} \quad f_{n}=\lambda_{n} / \lambda_{t} .
$$

We assume the porous media domain $\Omega \subset \mathbb{R}^{d}(d=2,3)$ is a polyhedral bounded domain. Let $\Gamma=\partial \Omega$ be composed of $\Gamma_{D}$ and $\Gamma_{N}$ such that $\Gamma=\Gamma_{D} \cup \Gamma_{N}$ and $\Gamma_{D} \cap \Gamma_{N}=\emptyset$, where $\Gamma_{D}$ denotes the Dirichlet part of boundary and $\Gamma_{N}$ is the Neumann part of boundary. We also denote by $\Gamma_{\text {in }} \subset \Gamma$ the inflow boundary. We impose the initial and boundary conditions to the equations (2.1-2.4) as follows:

$$
\begin{array}{lll}
S_{\alpha}=S_{\alpha}^{0}, & t=0, & \\
p_{\alpha}=p_{\alpha}^{B}, & \text { on } \Gamma_{D}, & \alpha=w, n, \\
\boldsymbol{u}_{\alpha} \cdot \boldsymbol{n}=g_{\alpha}^{N}, & \text { on } \Gamma_{N}, & \alpha=w, n, \\
S_{\alpha}=S_{\alpha}^{B}, & \text { on } \Gamma_{\mathrm{in}}, & \alpha=w, n .
\end{array}
$$

We also assume that the absolute permeability tensor $\boldsymbol{K}$ is symmetric positive and definite which can be homogeneous or heterogeneous, the porosity $\phi$ is time-independent and uniformly bounded below and above, and there exist positive constants $\lambda_{w}^{a}, \lambda_{n}^{a}, \lambda_{t}^{a}$ such that the mobilities are satisfying $0 \leq \lambda_{w}\left(S_{w}\right) \leq \lambda_{w}^{a}$, $0 \leq \lambda_{n}\left(S_{w}\right) \leq \lambda_{n}^{a}, 0<\lambda_{t}\left(S_{w}\right) \leq \lambda_{t}^{a}$.

In the homogeneous porous media, the standard IMPES scheme $[45,46]$ is widely used to simulate the above two-phase flow. The conservation law of total volume is directly obtained by the summation of the conservation laws of each phase and the constraint of the saturations of phases (2.3) as follows:

$$
\nabla \cdot \boldsymbol{u}_{t}=F_{t}
$$

where $\boldsymbol{u}_{t}=\boldsymbol{u}_{w}+\boldsymbol{u}_{n}$ is the total Darcy velocity and $F_{t}=F_{w}+F_{n}$ is the total external mass flow rate. By the Darcy's law of each phase (2.2) and the equation of capillary pressure (2.4), one can rewrite the total Darcy velocity into

$$
\boldsymbol{u}_{t}=-\lambda_{t} \boldsymbol{K} \nabla p_{w}-\lambda_{n} \boldsymbol{K} \nabla p_{c}-\left(\lambda_{w} \rho_{w}+\lambda_{n} \rho_{n}\right) \boldsymbol{K} g \nabla z .
$$

Then the conservation law of total volume can also be rewritten into

$$
-\nabla \cdot\left(\lambda_{t} \boldsymbol{K} \nabla p_{w}\right)=F_{t}+\nabla \cdot\left(\lambda_{n} \boldsymbol{K} \nabla p_{c}\right)+\nabla \cdot\left(\left(\lambda_{w} \rho_{w}+\lambda_{n} \rho_{n}\right) \boldsymbol{K} g \nabla z\right):=\operatorname{RHS}_{p r e s}\left(S_{w}\right),
$$

which is known as the pressure equation for two-phase flow. For given $S_{w}$, the pressure equation is linear with respect to the wetting phase pressure $p_{w}$. For the wetting phase, substituting $\boldsymbol{u}_{w}=f_{w} \boldsymbol{u}_{t}+\lambda_{n} f_{w} \boldsymbol{K} \nabla p_{c}-$ 
$\lambda_{n} f_{w}\left(\rho_{w}-\rho_{n}\right) \boldsymbol{K} g \nabla z$ into the wetting phase conservation law and noting that $\nabla p_{c}=\frac{d p_{c}\left(S_{w}\right)}{d S_{w}} \nabla S_{w}$, we get the following saturation equation:

$$
\phi \frac{\partial S_{w}}{\partial t}=F_{w}-\nabla \cdot\left(f_{w} \boldsymbol{u}_{t}\right)-\nabla \cdot\left(\lambda_{n} f_{w} \boldsymbol{K}\left(\frac{d p_{c}}{d S_{w}} \nabla S_{w}-\left(\rho_{w}-\rho_{n}\right) g \nabla z\right)\right):=\operatorname{RHS}_{s a t}\left(p_{w}, S_{w}\right),
$$

which is known as the saturation equation. Then for given $S_{w}^{n}$ from the time step $n$, the standard IMPES scheme implicitly solves $p_{w}^{n+1}$ by the pressure equation (2.10) and explicitly updates the saturation $S_{w}^{n+1}$ of wetting phase by the saturation equation (2.11) at the time step $n+1$ as follows:

$$
\begin{aligned}
-\nabla \cdot\left(\lambda_{t}\left(S_{w}^{n}\right) \boldsymbol{K} \nabla p_{w}^{n+1}\right) & =\operatorname{RHS}_{s a t}\left(S_{w}^{n}\right), \\
\phi \frac{S_{w}^{n+1}-S_{w}^{n}}{t_{n+1}-t_{n}} & =\operatorname{RHS}_{s a t}\left(p_{w}^{n+1}, S_{w}^{n}\right) .
\end{aligned}
$$

In the heterogeneous porous media, the capillary discontinuity may usually arise from contrast in capillary pressure functions, and in this case, the saturation is discontinuous due to capillary continuity. For different capillary pressure functions, the above standard IMPES scheme does not reproduce the correct saturation solution with discontinuity. In [27, 28], Hoteit and Firoozabadi proposed a revised HF-IMPES scheme to treat contrast in capillary pressure of heterogeneous porous media, which takes the advantage of the fact that the total mobility $\lambda_{t}$ is smoother than the wetting-phase mobility $\lambda_{w}$.

Define $\Phi_{\alpha}=p_{\alpha}+\rho_{\alpha} g z, \alpha=w, n$ and $\Phi_{c}=\Phi_{n}-\Phi_{w}=p_{c}+\left(\rho_{n}-\rho_{w}\right) g z$. The total velocity $\boldsymbol{u}_{t}$ is defined as the sum of two velocity variables $\boldsymbol{u}_{a}$ and $\boldsymbol{u}_{c}$ as $\boldsymbol{u}_{t}=\boldsymbol{u}_{a}+\boldsymbol{u}_{c}$, where $\boldsymbol{u}_{a}=-\lambda_{t} \boldsymbol{K} \nabla \Phi_{w}$ and $\boldsymbol{u}_{c}=-\lambda_{n} \boldsymbol{K} \nabla \Phi_{c}$. We note that the velocity $\boldsymbol{u}_{a}$ has the same driving force as the wetting-phase velocity but with a smoother mobility $\lambda_{t}$ than the wetting phase mobility. Then the HF-IMPES scheme consists of three steps as follows:

Step 1. Given $S_{w}^{n}$, seek $\boldsymbol{u}_{c}^{n+1}$ such that

$$
\boldsymbol{u}_{c}^{n+1}=-\lambda_{n}\left(S_{w}^{n}\right) \boldsymbol{K} \nabla \Phi_{c}\left(S_{w}^{n}\right) .
$$

Step 2. Given $S_{w}^{n}$ and $\boldsymbol{u}_{c}^{n+1}$, seek $\boldsymbol{u}_{a}^{n+1}$ and $\Phi_{w}^{n+1}$ by

$$
\nabla \cdot \boldsymbol{u}_{a}^{n+1}=F_{t}-\nabla \cdot \boldsymbol{u}_{c}^{n+1}, \quad \boldsymbol{u}_{a}^{n+1}=-\lambda_{t}\left(S_{w}^{n}\right) \boldsymbol{K} \nabla \Phi_{w}^{n+1} .
$$

Step 3. Given $S_{w}^{n}, \boldsymbol{u}_{a}^{n+1}$ and $\Phi_{w}^{n+1}$, update the wetting and non-wetting phase saturations by

$$
\phi \frac{S_{w}^{n+1}-S_{w}^{n}}{t_{n+1}-t_{n}}=F_{w}-\nabla \cdot\left(f_{w}\left(S_{w}^{n}\right) \boldsymbol{u}_{a}^{n+1}\right), \quad S_{n}^{n+1}=1-S_{w}^{n+1} .
$$

Like the standard IMPES scheme, the HF-IMPES scheme is only locally mass-conservative for the wetting phase. Consequently, both of the two schemes are not locally mass-conservative for the total fluid mixture. Besides, both of the two schemes are biased and might produce a wetting-phase saturation that larger than one. In the following section, we will introduce two new fully mass-conservative IMPES scheme to solve the two-phase flow problem (2.1-2.9) in porous media.

\section{Fully MASS-CONSERVATIVE IMPES SCHEMES}

In this section we present two fully mass-conservative IMPES schemes for incompressible and immiscible two-phase flow problem (2.1-2.9) in porous media. We begin with some notations. We use the standard notations and definitions for Sobolev spaces (cf. [2]) throughout the paper. For any $D \subset \bar{\Omega}$, any scalar functions $\psi$ and $\phi$, or vector functions $\boldsymbol{\psi}$ and $\boldsymbol{\phi}$, we define $(\psi, \phi)_{D}=\int_{D} \psi \phi$ and $(\boldsymbol{\psi}, \boldsymbol{\phi})_{D}=\int_{D} \boldsymbol{\psi} \cdot \boldsymbol{\phi}$. When $D=\Omega$, we use the notation $\|\cdot\|_{0, D}$ to denote the $L^{2}$-norm on $D$. We denote by $\mathcal{T}_{h}$ the quasi-uniform grid on $\Omega, \mathcal{E}_{h}$ the set of all faces $(d=3)$ or edges $(d=2)$ of $\mathcal{T}_{h}, h_{K}$ the diameter of any element $K \in \mathcal{T}_{h}$, $h=\min _{K \in \mathcal{T}_{h}} h_{K}$. Let $K, K^{\prime} \in \mathcal{T}_{h}$ and $F=\partial K \cap \partial K^{\prime}$ with the outward unit normal vector $\boldsymbol{n}_{F}$ exterior to $K$. We denote $\llbracket \psi \rrbracket=\left.\left(\left.\psi\right|_{K}\right)\right|_{F}-\left.\left(\left.\psi\right|_{K^{\prime}}\right)\right|_{F}$ the jump of scalar function $\psi$ across interior edges/faces $F$, $\{\psi\}=\frac{1}{2}\left(\left.\left(\left.\psi\right|_{K}\right)\right|_{F}+\left.\left(\left.\psi\right|_{K^{\prime}}\right)\right|_{F}\right)$ the average of scalar function $\psi$ across interior edges/faces $F$, and $\llbracket \psi \rrbracket,\{\psi\}$ denote $\psi$ on edges/faces on $\partial \Omega$. We denote by $C$ with or without subscript a positive constant depending on the shape regularity of the meshes and the coefficients data in (2.1-2.4). These constants can take on different values in different occurrences.

Next, we introduce the lowest-order Raviart-Thomas mixed finite element approximation which will be used in the following spacial discrete schemes for simplicity. Let $R T_{0}$ be the well-known lowest-order of 
Raviart-Thomas finite element space. On the simplicial mesh, $R T_{0}=\left[P_{0}\right]^{d}+\boldsymbol{x} P_{0}$, where $P_{0}$ is the piecewise constant space. We define

$$
\begin{aligned}
\boldsymbol{U}_{h} & =\left\{\boldsymbol{v}_{h} \in H(\operatorname{div}, \Omega):\left.v_{h}\right|_{K} \in R T_{0}(K), \forall K \in \mathcal{T}_{h}\right\}, \\
Q_{h} & =\left\{q_{h} \in L^{2}(\Omega):\left.q_{h}\right|_{K} \in P_{0}(K), \forall K \in \mathcal{T}_{h}\right\},
\end{aligned}
$$

where $H(\operatorname{div}, \Omega)=\left\{\boldsymbol{v} \in\left[L^{2}(\Omega)\right]^{d}: \nabla \cdot \boldsymbol{v} \in L^{2}(\Omega)\right\}$. Let $\boldsymbol{U}_{h}^{0}=\left\{\boldsymbol{v} \in \boldsymbol{U}_{h}: \boldsymbol{v} \cdot \boldsymbol{n}=0\right.$ on $\left.\Gamma_{N}\right\}$. In the following we will approximate the pressure-velocity systems by the lowest-order Raviart-Thomas mixed finite element method with upwind scheme. When we use the lowest-order Raviart-Thomas mixed finite element method on structured grid, we note that the mixed finite element method is equivalent to the cell-centered finite difference method on structured grid if we apply the trapezoidal rule for integration in the mixed finite element method.

3.1. A fully mass-conservative IMPES scheme of the first kind (FC-IMPES-I). We first introduce the FC-IMPES-I scheme. Define $\boldsymbol{w}_{\alpha}=-\boldsymbol{K}\left(\nabla p_{\alpha}+\rho_{\alpha} g \nabla z\right)$, we have $\boldsymbol{u}_{\alpha}=\lambda_{\alpha} \boldsymbol{w}_{\alpha}$. We assume $\boldsymbol{w}_{\alpha} \in H(\operatorname{div}, \Omega)$, $p_{\alpha} \in L^{2}(\Omega)$ and $S_{\alpha} \in L^{2}(\Omega)$. For any $\boldsymbol{v}_{\alpha} \in \boldsymbol{U}_{h}, q \in Q_{h}$ and $S_{w}^{h} \in Q_{h}$, we define a bilinear formulation $B_{\alpha}\left(\boldsymbol{v}_{\alpha}, q ; S_{w}^{h}\right)$ as follows:

$$
B_{\alpha}\left(\boldsymbol{v}_{\alpha}, q ; S_{w}^{h}\right)=\left(\nabla \cdot\left(\lambda_{\alpha}\left(S_{w}^{h}\right) \boldsymbol{v}_{\alpha}\right), q\right)-\sum_{K \in \mathcal{T}_{h}} \int_{\partial K_{\alpha}^{-} \backslash \Gamma} \llbracket \lambda_{\alpha}\left(S_{w}^{h}\right) \rrbracket \boldsymbol{v}_{\alpha} \cdot \boldsymbol{n} q,
$$

where $\partial K_{\alpha}^{-}=\left\{e \subset \partial K:\left.\left\{\boldsymbol{u}_{\alpha}^{h} \cdot \boldsymbol{n}_{e}\right\}\right|_{e}<0\right\}$ with the normal vector $n_{e}$ exterior to $K$. Here, $\boldsymbol{u}_{\alpha}^{h}$ is the discrete velocity of phase $\alpha$. Actually, this is an upwind scheme for $\lambda_{\alpha}\left(S_{w}^{h}\right)$ on $\partial K$. Indeed, if $q \in Q_{h}$ is piecewise constant, we can compute $B_{\alpha}\left(\boldsymbol{v}_{\alpha}, q ; S_{w}^{h}\right)$ as follows:

$$
\begin{aligned}
B_{\alpha}\left(\boldsymbol{v}_{\alpha}, q ; S_{w}^{h}\right) & =\sum_{K \in \mathcal{T}_{h}} \int_{\partial K} \lambda_{\alpha}\left(S_{w}^{h}\right) \boldsymbol{v}_{\alpha} \cdot \boldsymbol{n} q-\sum_{K \in \mathcal{T}_{h}} \int_{\partial K_{\alpha}^{-} \backslash \Gamma} \llbracket \lambda_{\alpha}\left(S_{w}^{h}\right) \rrbracket \boldsymbol{v}_{\alpha} \cdot \boldsymbol{n} q \\
& =\sum_{K \in \mathcal{T}_{h}} \int_{\partial K} \lambda_{\alpha}\left(S_{w, \alpha}^{*, h}\right) \boldsymbol{v}_{\alpha} \cdot \boldsymbol{n} q,
\end{aligned}
$$

where the upwind value $S_{w, \alpha}^{*, h}$ in the function $\lambda_{\alpha}\left(S_{w}^{h}\right)$ is defined as follows:

$$
S_{\alpha}^{*, h}=\left\{\begin{array}{ll}
\left.S_{\alpha}^{h}\right|_{K_{i}}, & \text { if }\left\{\lambda_{\alpha}\left(S_{w}^{h}\right) \boldsymbol{v}_{\alpha} \cdot \boldsymbol{n}_{\gamma}\right\}_{\gamma} \geq 0, \\
\left.S_{\alpha}^{h}\right|_{K_{j}}, & \text { if }\left\{\lambda_{\alpha}\left(S_{w}^{h}\right) \boldsymbol{v}_{\alpha} \cdot \boldsymbol{n}_{\gamma}\right\}_{\gamma}<0,
\end{array} \quad S_{w, \alpha}^{*, h}= \begin{cases}S_{w}^{*, h}, & \alpha=w, \\
1-S_{n}^{*, h}, & \alpha=n .\end{cases}\right.
$$

Here we denote $\gamma=\partial K_{i} \cap \partial K_{j}$ with the normal vector $\boldsymbol{n}_{\gamma}$ exterior to $K_{i}$. If $\gamma \subset \Gamma_{\text {in }}$, then $\left.S_{w, \alpha}^{* h}\right|_{\gamma}=\left.P_{\gamma} S_{w}^{B}\right|_{\gamma}$, where $P_{\gamma}$ is the $L^{2}$-projection operator into $P_{0}(\gamma)$. In the following, we always assume that for any $\bar{S}_{w} \in Q_{h}$, there holds

$$
\left.\lambda_{w}\left(\bar{S}_{w, w}^{*}\right)\right|_{F}+\left.\lambda_{n}\left(\bar{S}_{w, n}^{*}\right)\right|_{F} \geq \lambda_{0}>0,
$$

the upwind values in $\lambda_{\alpha}\left(\bar{S}_{w, \alpha}^{*}\right), \alpha=n, w$, are determined by some known phase velocity.

Let $J=(0, T]$, now we have the following continuous-in-time and discrete-in-space nonlinear system to solve the two-phase flow problem (2.1-2.9) in porous media. For any $\boldsymbol{v} \in \boldsymbol{U}_{h}^{0}$ and $q \in Q_{h}$, we find $\boldsymbol{w}_{\alpha}^{h}(\cdot, t) \in \boldsymbol{U}_{h}, p_{\alpha}^{h}(\cdot, t) \in Q_{h}, S_{\alpha}^{h}(\cdot, t) \in Q_{h}, \alpha=w, n$, such that

$$
\begin{aligned}
& \left(\phi \frac{\partial S_{\alpha}^{h}}{\partial t}, q\right)+B_{\alpha}\left(\boldsymbol{w}_{\alpha}^{h}, q ; S_{w}^{h}\right)=\left(F_{\alpha}, q\right), \quad \alpha=w, n, t \in J, \\
& \left(\boldsymbol{K}^{-1} \boldsymbol{w}_{\alpha}^{h}, \boldsymbol{v}\right)-\left(p_{\alpha}^{h}, \nabla \cdot \boldsymbol{v}\right)=-\int_{\Gamma_{D}} p_{\alpha}^{B} \boldsymbol{v} \cdot \boldsymbol{n}-\left(\rho_{\alpha} g \nabla z, \boldsymbol{v}\right), \quad \alpha=w, n, t \in J, \\
& S_{n}^{h}+S_{w}^{h}=1, \quad t \in J, \\
& \left(p_{n}^{h}-p_{w}^{h}, q\right)=\left(p_{c}\left(S_{w}^{h}\right), q\right), \quad t \in J, \\
& \left(S_{w}^{h}, q\right)=\left(S_{w}^{0}, q\right), \quad t=0 .
\end{aligned}
$$


Let $S_{w}^{h, n} \in Q_{h}$ be given at the time step $n$, then we find $\boldsymbol{w}_{\alpha}^{h, n+1} \in \boldsymbol{U}_{h}, p_{\alpha}^{h, n+1} \in Q_{h}, S_{\alpha}^{h, n+1} \in Q_{h}$, $\alpha=w, n$ at the next time step $n+1$ as follows:

$$
\begin{array}{ll}
\left(\phi \frac{S_{\alpha}^{h, n+1}-S_{\alpha}^{h, n}}{t_{n+1}-t_{n}}, q\right)+B_{\alpha}\left(\boldsymbol{w}_{\alpha}^{h, n+1}, q ; S_{w}^{h, n}\right)=\left(F_{\alpha}, q\right), & \alpha=w, n, \\
\left(\boldsymbol{K}^{-1} \boldsymbol{w}_{\alpha}^{h, n+1}, \boldsymbol{v}\right)-\left(p_{\alpha}^{h, n+1}, \nabla \cdot \boldsymbol{v}\right)=-\int_{\Gamma_{D}} p_{\alpha}^{B} \boldsymbol{v} \cdot \boldsymbol{n}-\left(\rho_{\alpha} g \nabla z, \boldsymbol{v}\right), & \alpha=w, n, \\
S_{n}^{h, n+1}+S_{w}^{h, n+1}=1, & \\
\left(p_{n}^{h, n+1}-p_{w}^{h, n+1}, q\right)=\left(p_{c}\left(S_{w}^{h, n}\right), q\right) . &
\end{array}
$$

By summing the above discrete conservation law for each phase and noting the constraint of the saturations of phases, we now solve the following linear system to seek $\boldsymbol{w}_{\alpha}^{h, n+1} \in \boldsymbol{U}_{h}$ and $p_{\alpha}^{h, n+1} \in Q_{h}$ such that

$$
\begin{aligned}
& \sum_{\alpha} B_{\alpha}\left(\boldsymbol{w}_{\alpha}^{h, n+1}, q ; S_{w}^{h, n}\right)=\left(F_{t}, q\right), \\
& \left(\boldsymbol{K}^{-1} \boldsymbol{w}_{\alpha}^{h, n+1}, \boldsymbol{v}\right)-\left(p_{\alpha}^{h, n+1}, \nabla \cdot \boldsymbol{v}\right)=-\int_{\Gamma_{D}} p_{\alpha}^{B} \boldsymbol{v} \cdot \boldsymbol{n}-\left(\rho_{\alpha} g \nabla z, \boldsymbol{v}\right), \quad \alpha=w, n, \\
& \left(p_{n}^{h, n+1}-p_{w}^{h, n+1}, q\right)=\left(p_{c}\left(S_{w}^{h, n}\right), q\right) .
\end{aligned}
$$

Define $\boldsymbol{w}_{c}^{h, n+1}=\boldsymbol{w}_{n}^{h, n+1}-\boldsymbol{w}_{w}^{h, n+1}$. For any $\boldsymbol{v} \in \boldsymbol{U}_{h}^{0}$, we have $\nabla \cdot \boldsymbol{v} \in Q_{h}$. Thus, by (3.9) and (3.10), we have

$$
\left(\boldsymbol{K}^{-1} \boldsymbol{w}_{c}^{h, n+1}, \boldsymbol{v}\right)=\left(p_{c}\left(S_{w}^{h, n}\right), \nabla \cdot \boldsymbol{v}\right)-\int_{\Gamma_{D}}\left(p_{n}^{B}-p_{w}^{B}\right) \boldsymbol{v} \cdot \boldsymbol{n}-\left(\left(\rho_{n}-\rho_{w}\right) g \nabla z, \boldsymbol{v}\right) .
$$

It is easy to see that (3.11) is well-posed for the solution of $\boldsymbol{w}_{c}^{h, n+1}$. Then the solution of the linear system (3.8-3.10) can be decoupled into two steps. Firstly, we can solve (3.10) and (3.11) to get $p_{n}^{h, n+1}-p_{w}^{h, n+1}$ and $\boldsymbol{w}_{c}^{h, n+1}$. Next, $p_{w}^{h, n+1}$ and $\boldsymbol{w}_{w}^{h, n+1}$ can be obtained by (3.8) and (3.9), then $p_{n}^{h, n+1}$ and $\boldsymbol{w}_{n}^{h, n+1}$ are directly obtained. For any $(\boldsymbol{v}, q) \in \boldsymbol{U}_{h} \times Q_{h}$, we define $B_{t}\left(\boldsymbol{v}, q ; S_{w}^{h, n}\right)=\sum_{\alpha} B_{\alpha}\left(\boldsymbol{v}, q ; S_{w}^{h, n}\right)$. Now we present the FC-IMPES-I scheme.

Algorithm 3.1. (FC-IMPES-I scheme) Given $S_{w}^{h, n}$ at the time step $n$, we seek the solutions of the linear system (3.4-3.7) at the time step $n+1$ as follows:

Step 1. Seek $p_{n}^{h, n+1}-p_{w}^{h, n+1} \in Q_{h}$ and $\boldsymbol{w}_{c}^{h, n+1} \in \boldsymbol{U}_{h}$ by (3.10) and (3.11) respectively.

Step 2. Seek $p_{w}^{h, n+1}$ and $\boldsymbol{w}_{w}^{h, n+1}$ by

$$
\begin{aligned}
& B_{t}\left(\boldsymbol{w}_{w}^{h, n+1}, q ; S_{w}^{h, n}\right)=\left(F_{t}, q\right)-B_{n}\left(\boldsymbol{w}_{c}^{h, n+1}, q ; S_{w}^{h, n}\right), \quad q \in Q_{h}, \\
& \left(\boldsymbol{K}^{-1} \boldsymbol{w}_{w}^{h, n+1}, \boldsymbol{v}\right)-\left(p_{w}^{h, n+1}, \nabla \cdot \boldsymbol{v}\right)=-\int_{\Gamma_{D}} p_{w}^{B} \boldsymbol{v} \cdot \boldsymbol{n}-\left(\rho_{w} g \nabla z, \boldsymbol{v}\right), \quad \boldsymbol{v} \in \boldsymbol{U}_{h}^{0} .
\end{aligned}
$$

Then $p_{n}^{h, n+1}$ and $\boldsymbol{w}_{n}^{h, n+1}$ can be updated by

$$
p_{n}^{h, n+1}=\left(p_{n}^{h, n+1}-p_{w}^{h, n+1}\right)+p_{w}^{h, n+1}, \quad \boldsymbol{w}_{n}^{h, n+1}=\boldsymbol{w}_{c}^{h, n+1}+\boldsymbol{w}_{w}^{h, n+1} .
$$

Step 3. We can update the phase saturation $S_{\alpha}^{h, n+1}, \alpha=w, n$ by the following two approaches.

Approach I: Update the wetting phase saturation $S_{w}^{h, n+1}$ by

$$
\left(\phi \frac{S_{w}^{h, n+1}-S_{w}^{h, n}}{t_{n+1}-t_{n}}, q\right)+B_{w}\left(\boldsymbol{w}_{w}^{h, n+1}, q ; S_{w}^{h, n}\right)=\left(F_{w}, q\right) .
$$

Then the non-wetting phase saturation $S_{n}^{h, n+1}$ is updated by $S_{n}^{h, n+1}=1-S_{w}^{h, n+1}$.

Approach II: Update the wetting and non-wetting phase saturations $S_{w}^{h, n+1}, S_{n}^{h, n+1}$ respectively by

$$
\begin{aligned}
& \left(\phi \frac{S_{w}^{h, n+1}-S_{w}^{h, n}}{t_{n+1}-t_{n}}, q\right)+B_{w}\left(\boldsymbol{w}_{w}^{h, n+1}, q ; S_{w}^{h, n}\right)=\left(F_{w}, q\right), \\
& \left(\phi \frac{S_{n}^{h, n+1}-S_{n}^{h, n}}{t_{n+1}-t_{n}}, q\right)+B_{n}\left(\boldsymbol{w}_{n}^{h, n+1}, q ; S_{w}^{h, n}\right)=\left(F_{n}, q\right) .
\end{aligned}
$$


Remark 3.1. We note that (3.13) and (3.11) directly yield that

$$
\left(\boldsymbol{K}^{-1} \boldsymbol{w}_{n}^{h, n+1}, \boldsymbol{v}\right)-\left(p_{n}^{h, n+1}, \nabla \cdot \boldsymbol{v}\right)=-\int_{\Gamma_{D}} p_{n}^{B} \boldsymbol{v} \cdot \boldsymbol{n}-\left(\rho_{n} g \nabla z, \boldsymbol{v}\right), \quad \boldsymbol{v} \in \boldsymbol{U}_{h}^{0} .
$$

When (3.12) holds, it also yields

$$
B_{t}\left(\boldsymbol{w}_{n}^{h, n+1}, q ; S_{w}^{h, n}\right)=\left(F_{t}, q\right)+B_{w}\left(\boldsymbol{w}_{c}^{h, n+1}, q ; S_{w}^{h, n}\right), \quad q \in Q_{h} .
$$

Thus we can see that Algorithm 3.1 is unbiased in the solution of the auxiliary velocity $\boldsymbol{w}_{\alpha}$ and pressure $p_{\alpha}$ for each phase.

Lemma 3.2. If $S_{w}^{h, n}+S_{n}^{h, n}=1$, then two approaches in Step 3 of the FC-IMPES-I scheme are equivalent.

Proof. On one hand, if we choose Approach II in Step 3 of the FC-IMPES-I scheme, then (3.15) and (3.16) both hold true. Due to the fact that (3.8) has been used in the computation, we directly have that

$$
\left(\phi \frac{S_{w}^{h, n+1}-S_{w}^{h, n}}{t_{n+1}-t_{n}}, q\right)+\left(\phi \frac{S_{n}^{h, n+1}-S_{n}^{h, n}}{t_{n+1}-t_{n}}, q\right)=0, \quad q \in Q_{h}
$$

which, together with the assumption $S_{w}^{h, n}+S_{n}^{h, n}=1$, yields that

$$
S_{w}^{h, n+1}+S_{n}^{h, n+1}=1 \text {. }
$$

On the other hand, if we choose Approach I in Step 3 of the FC-IMPES-I scheme, by (3.14) and the facts $S_{w}^{h, k}+S_{n}^{h, k}=1, k=n, n+1$ and (3.8), we directly observe that (3.16) holds true.

Remark 3.3. The solutions of the wetting phase saturation in standard IMPES and HF-IMPES schemes are similar to the Approach I in Step 3 of the FC-IMPES-I scheme. Since the mass-conservation property only holds true for one phase in standard IMPES and HF-IMPES schemes, the similar two approaches as the above Approaches I and II in standard IMPES and HF-IMPES schemes are not always equivalent. However, from Lemma 3.2, we can see that the two approaches in Step 3 of the FC-IMPES-I scheme are equivalent and the mass-conservative property holds true for both phases.

3.2. A fully mass-conservative IMPES scheme of the second kind (FC-IMPES-II). Next, we introduce the FC-IMPES-II scheme. Define $\boldsymbol{\xi}_{\alpha}=\lambda_{t} \boldsymbol{w}_{\alpha}$ with $\boldsymbol{w}_{\alpha}=-\boldsymbol{K}\left(\nabla p_{\alpha}+\rho_{\alpha} g \nabla z\right)$. Then we easily have $\boldsymbol{u}_{\alpha}=f_{\alpha} \boldsymbol{\xi}_{\alpha}$ where $f_{\alpha}$ is the fractional flow function defined in (2.5). We assume $\boldsymbol{\xi}_{\alpha} \in H(\operatorname{div}, \Omega), p_{\alpha} \in L^{2}(\Omega)$ and $S_{\alpha} \in L^{2}(\Omega)$. For any $\boldsymbol{v}_{\alpha} \in \boldsymbol{U}_{h}, q \in Q_{h}$ and $S_{w}^{h} \in Q_{h}$, we define

$$
\widetilde{B}_{\alpha}\left(\boldsymbol{v}_{\alpha}, q ; S_{w}^{h}\right)=\left(\nabla \cdot\left(f_{\alpha}\left(S_{w}^{h}\right) \boldsymbol{v}_{\alpha}\right), q\right)-\sum_{K \in \mathcal{T}_{h}} \int_{\partial K_{\alpha}^{-} \backslash \Gamma} \llbracket f_{\alpha}\left(S_{w}^{h}\right) \rrbracket \boldsymbol{v}_{\alpha} \cdot \boldsymbol{n} q .
$$

Here $\partial K_{\alpha}^{-}$is defined as in Section 3.1, and the above bilinear formulation is also an upwind scheme for $f_{\alpha}\left(S_{w}^{h}\right)$ on $\partial K$. Indeed, $\widetilde{B}_{\alpha}\left(\boldsymbol{v}_{\alpha}, q ; S_{w}^{h}\right)$ can be rewritten as follows:

$$
\widetilde{B}_{\alpha}\left(\boldsymbol{v}_{\alpha}, q ; S_{w}^{h}\right)=\sum_{K \in \mathcal{T}_{h}} \int_{\partial K} f_{\alpha}\left(S_{w, \alpha}^{*, h}\right) \boldsymbol{v}_{\alpha} \cdot \boldsymbol{n} q,
$$

where the upwind value $S_{w, \alpha}^{*, h}$ in the function $f_{\alpha}\left(S_{w, \alpha}^{*, h}\right)$ is defined as in the function $\lambda_{\alpha}\left(S_{w, \alpha}^{*, h}\right)$ in (3.2).

Let $J=(0, T]$, we have the following continuous-in-time and discrete-in-space nonlinear system to solve the two-phase flow problem (2.1-2.9) in porous media. For any $\boldsymbol{v} \in \boldsymbol{U}_{h}^{0}$ and $q \in Q_{h}$, we find $\boldsymbol{\xi}_{\alpha}^{h}(\cdot, t) \in \boldsymbol{U}_{h}$, $p_{\alpha}^{h}(\cdot, t) \in Q_{h}, S_{\alpha}^{h}(\cdot, t) \in Q_{h}, \alpha=w, n$, such that

$$
\begin{aligned}
& \left(\phi \frac{\partial S_{\alpha}^{h}}{\partial t}, q\right)+\widetilde{B}_{\alpha}\left(\boldsymbol{\xi}_{\alpha}^{h}, q ; S_{w}^{h}\right)=\left(F_{\alpha}, q\right), \quad \alpha=w, n, t \in J, \\
& \left(\left(\lambda_{t} \boldsymbol{K}\right)^{-1} \boldsymbol{\xi}_{\alpha}^{h}, \boldsymbol{v}\right)-\left(p_{\alpha}^{h}, \nabla \cdot \boldsymbol{v}\right)=-\int_{\Gamma_{D}} p_{\alpha}^{B} \boldsymbol{v} \cdot \boldsymbol{n}-\left(\rho_{\alpha} g \nabla z, \boldsymbol{v}\right), \quad \alpha=w, n, t \in J, \\
& S_{n}^{h}+S_{w}^{h}=1, \quad t \in J, \\
& \left(p_{n}^{h}-p_{w}^{h}, q\right)=\left(p_{c}\left(S_{w}^{h}\right), q\right), \quad t \in J, \\
& \left(S_{w}^{h}, q\right)=\left(S_{w}^{0}, q\right), \quad t=0 .
\end{aligned}
$$


We assume the above nonlinear system is also well-posed. Let $S_{w}^{h, n} \in Q_{h}$ be given at the time step $n$, then we find $\boldsymbol{\xi}_{\alpha}^{h, n+1} \in \boldsymbol{U}_{h}, p_{\alpha}^{h, n+1} \in Q_{h}, S_{\alpha}^{h, n+1} \in Q_{h}, \alpha=w, n$ at the next time step $n+1$ as follows:

$$
\begin{array}{ll}
\left(\phi \frac{S_{\alpha}^{h, n+1}-S_{\alpha}^{h, n}}{t_{n+1}-t_{n}}, q\right)+\widetilde{B}_{\alpha}\left(\boldsymbol{\xi}_{\alpha}^{h, n+1}, q ; S_{w}^{h, n}\right)=\left(F_{\alpha}, q\right), & \alpha=w, n, \\
\left(\left(\lambda_{t} \boldsymbol{K}\right)^{-1} \boldsymbol{\xi}_{\alpha}^{h, n+1}, \boldsymbol{v}\right)-\left(p_{\alpha}^{h, n+1}, \nabla \cdot \boldsymbol{v}\right)=-\int_{\Gamma_{D}} p_{\alpha}^{B} \boldsymbol{v} \cdot \boldsymbol{n}-\left(\rho_{\alpha} g \nabla z, \boldsymbol{v}\right), & \alpha=w, n, \\
S_{n}^{h, n+1}+S_{w}^{h, n+1}=1, & \\
\left(p_{n}^{h, n+1}-p_{w}^{h, n+1}, q\right)=\left(p_{c}\left(S_{w}^{h, n}\right), q\right) . &
\end{array}
$$

We can compute $\widetilde{B}_{\alpha}\left(\boldsymbol{\xi}_{\alpha}^{h, n+1}, q ; S_{w}^{h, n}\right)$ as in (3.1). Similar to (3.8-3.10), we seek $\boldsymbol{\xi}_{\alpha}^{h, n+1} \in \boldsymbol{U}_{h}, p_{\alpha}^{h, n+1} \in Q_{h}$ such that

$$
\begin{aligned}
& \sum_{\alpha} \widetilde{B}_{\alpha}\left(\boldsymbol{\xi}_{\alpha}^{h, n+1}, q ; S_{w}^{h, n}\right)=\left(F_{t}, q\right) \\
& \left(\left(\lambda_{t} \boldsymbol{K}\right)^{-1} \boldsymbol{\xi}_{\alpha}^{h, n+1}, \boldsymbol{v}\right)-\left(p_{\alpha}^{h, n+1}, \nabla \cdot \boldsymbol{v}\right)=-\int_{\Gamma_{D}} p_{\alpha}^{B} \boldsymbol{v} \cdot \boldsymbol{n}-\left(\rho_{\alpha} g \nabla z, \boldsymbol{v}\right), \quad \alpha=w, n, \\
& \left(p_{n}^{h, n+1}-p_{w}^{h, n+1}, q\right)=\left(p_{c}\left(S_{w}^{h, n}\right), q\right) .
\end{aligned}
$$

Define $\boldsymbol{\xi}_{c}^{h, n+1}=\boldsymbol{\xi}_{n}^{h, n+1}-\boldsymbol{\xi}_{w}^{h, n+1}$, by (3.23) we further have that

$$
\left(\left(\lambda_{t} \boldsymbol{K}\right)^{-1} \boldsymbol{\xi}_{c}^{h, n+1}, \boldsymbol{v}\right)=\left(p_{c}\left(S_{w}^{h, n}\right), \nabla \cdot \boldsymbol{v}\right)-\int_{\Gamma_{D}}\left(p_{n}^{B}-p_{w}^{B}\right) \boldsymbol{v} \cdot \boldsymbol{n}-\left(\left(\rho_{n}-\rho_{w}\right) g \nabla z, \boldsymbol{v}\right) .
$$

Similar to Algorithm 3.1, we can solve (3.23) and (3.24) to get $p_{n}^{h, n+1}-p_{w}^{h, n+1}$ and $\boldsymbol{\xi}_{c}^{h, n+1}$, then $p_{\alpha}^{h . n+1}$ and $\boldsymbol{\xi}_{\alpha}^{h, n+1}$ can be obtained by (3.21-3.22) by the similar approach as in Algorithm 3.1.

Given $\bar{S}_{w}$, for any $(\boldsymbol{v}, q) \in \boldsymbol{U}_{h} \times Q_{h}$, we define $\widetilde{B}_{t}\left(\boldsymbol{v}, q ; \bar{S}_{w}\right)=\sum_{\alpha} \widetilde{B}_{\alpha}\left(\boldsymbol{v}, q ; \bar{S}_{w}\right)$. Now we present the FC-IMPES-II scheme.

Algorithm 3.2. (FC-IMPES-II scheme) Given $S_{w}^{h, n}$ at the time step n, we seek the solutions of the linear system (3.17-3.20) at the time step $n+1$ as follows:

Step 1. Seek $p_{n}^{h, n+1}-p_{w}^{h, n+1} \in Q_{h}$ and $\boldsymbol{\xi}_{c}^{h, n+1} \in \boldsymbol{U}_{h}$ by (3.23) and (3.24) respectively.

Step 2. Seek $p_{w}^{h, n+1}$ and $\boldsymbol{\xi}_{w}^{h, n+1}$ by

$$
\begin{aligned}
& \widetilde{B}_{t}\left(\boldsymbol{\xi}_{w}^{h, n+1}, q ; S_{w}^{h, n}\right)=\left(F_{t}, q\right)-\widetilde{B}_{n}\left(\boldsymbol{\xi}_{c}^{h, n+1}, q ; S_{w}^{h, n}\right), \quad q \in Q_{h}, \\
& \left(\left(\lambda_{t} \boldsymbol{K}\right)^{-1} \boldsymbol{\xi}_{w}^{h, n+1}, \boldsymbol{v}\right)-\left(p_{w}^{h, n+1}, \nabla \cdot \boldsymbol{v}\right)=-\int_{\Gamma_{D}} p_{w}^{B} \boldsymbol{v} \cdot \boldsymbol{n}-\left(\rho_{w} g \nabla z, \boldsymbol{v}\right), \quad \boldsymbol{v} \in \boldsymbol{U}_{h}^{0} .
\end{aligned}
$$

Then $p_{n}^{h, n+1}$ and $\boldsymbol{\xi}_{n}^{h, n+1}$ can be updated by

$$
p_{n}^{h, n+1}=\left(p_{n}^{h, n+1}-p_{w}^{h, n+1}\right)+p_{w}^{h, n+1}, \quad \boldsymbol{\xi}_{n}^{h, n+1}=\boldsymbol{\xi}_{c}^{h, n+1}+\boldsymbol{\xi}_{w}^{h, n+1} .
$$

Step 3. We can update the phase saturation $S_{\alpha}^{h, n+1}, \alpha=w, n$ by the following two approaches.

Approach I: Update the wetting phase saturation $S_{w}^{h, n+1}$ by

$$
\left(\phi \frac{S_{w}^{h, n+1}-S_{w}^{h, n}}{t_{n+1}-t_{n}}, q\right)+\widetilde{B}_{w}\left(\boldsymbol{\xi}_{w}^{h, n+1}, q ; S_{w}^{h, n}\right)=\left(F_{w}, q\right) .
$$

Then the non-wetting phase saturation $S_{n}^{h, n+1}$ is updated by $S_{n}^{h, n+1}=1-S_{w}^{h, n+1}$.

Approach II: Update the wetting and non-wetting phase saturations $S_{w}^{h, n+1}, S_{n}^{h, n+1}$ respectively by

$$
\begin{aligned}
& \left(\phi \frac{S_{w}^{h, n+1}-S_{w}^{h, n}}{t_{n+1}-t_{n}}, q\right)+\widetilde{B}_{w}\left(\boldsymbol{\xi}_{w}^{h, n+1}, q ; S_{w}^{h, n}\right)=\left(F_{w}, q\right), \\
& \left(\phi \frac{S_{n}^{h, n+1}-S_{n}^{h, n}}{t_{n+1}-t_{n}}, q\right)+\widetilde{B}_{n}\left(\boldsymbol{\xi}_{n}^{h, n+1}, q ; S_{w}^{h, n}\right)=\left(F_{n}, q\right) .
\end{aligned}
$$

Similar to Lemma 3.2, we can also see that the two approaches in Step 3 of the FC-IMPES-II scheme are equivalent. Moreover, similar to Remark 3.1, we can easily see that Algorithm 3.2 is also unbiased in the solution of the auxiliary velocity $\boldsymbol{\xi}_{\alpha}$ and pressure $p_{\alpha}$ for each phase. 
Remark 3.4. When the capillary pressure and gravity effects are disregarded, we easily have that

$$
\boldsymbol{\xi}_{w}=-\lambda_{t} \boldsymbol{K} \nabla p_{w}=-\lambda_{t} \boldsymbol{K} \nabla p_{n}=\boldsymbol{\xi}_{n}, \quad \boldsymbol{u}_{t}=-\lambda_{t} \boldsymbol{K} \nabla p_{w}=-\lambda_{t} \boldsymbol{K} \nabla p_{n},
$$

and we see that $\boldsymbol{\xi}_{w}=\boldsymbol{\xi}_{n}=\boldsymbol{u}_{t}$. Thus, compared with Algorithm 3.1, the assumption $\boldsymbol{\xi}_{\alpha} \in H(\operatorname{div}, \Omega)$ in Algorithm 3.2 is more physical in some sense.

Remark 3.5. We remark that by the post-processing technique, the total velocity based on Algorithms 3.1 and 3.2 can be recovered to be continuous in the normal direction across the faces or edges of the elements in the whole domain. Actually, the total velocity in FC-IMPES-I scheme can be recovered as follows:

$$
\left.\boldsymbol{u}_{t}^{*, h, n+1} \cdot \boldsymbol{n}\right|_{F}=\left.\lambda_{w}\left(S_{w, w}^{*, h, n}\right) \boldsymbol{w}_{w}^{h, n+1} \cdot \boldsymbol{n}\right|_{F}+\left.\lambda_{n}\left(S_{w, n}^{*, h, n}\right) \boldsymbol{w}_{n}^{h, n+1} \cdot \boldsymbol{n}\right|_{F}, \quad F \in \mathcal{E}_{h} .
$$

Since $\boldsymbol{u}_{t}^{*, h, n+1} \in \boldsymbol{U}_{h}$, we can see that $\boldsymbol{u}_{t}^{*, h, n+1}$ is continuous in the normal direction across the faces or edges of the elements. Moreover, by (3.8) we can derive that

$$
\left(\nabla \cdot \boldsymbol{u}_{t}^{*, h, n+1}, q\right)=\left(F_{t}, q\right), \quad q \in Q_{h} .
$$

For the total velocity in FC-IMPES-II scheme, it can also be recovered by

$$
\left.\boldsymbol{u}_{t}^{*, h, n+1} \cdot \boldsymbol{n}\right|_{F}=\left.f_{w}\left(S_{w, w}^{*, h, n}\right) \boldsymbol{\xi}_{w}^{h, n+1} \cdot \boldsymbol{n}\right|_{F}+\left.f_{n}\left(S_{w, n}^{*, h, n}\right) \boldsymbol{\xi}_{n}^{h, n+1} \cdot \boldsymbol{n}\right|_{F}, \quad F \in \mathcal{E}_{h},
$$

and (3.27) also holds true for the above reconstructed total velocity.

\section{Analysis of THE NEW IMPES SCHEMES}

In this section we focus on the analysis of some merits of the FC-IMPES-I scheme. Similar analysis can also be shown for the FC-IMPES-II scheme.

4.1. Local mass conservation for both phases. For the FC-IMPES-I scheme, the approximate saturations of both phases satisfy the discrete mass-conservative law as in (3.4). For any $K \in \mathcal{T}_{h}$, taking $q=1$ in $\bar{K}$ and $q=0$ in $\Omega \backslash \bar{K}$ yields the following local mass conservation for both phases.

Lemma 4.1. For any $K \in \mathcal{T}_{h}$, the approximate saturations of both phases computed by the FC-IMPES-I scheme satisfy the following local mass-conservative property on $K$,

$$
\int_{K} \phi \frac{S_{\alpha}^{h, n+1}-S_{\alpha}^{h, n}}{t_{n+1}-t_{n}}+\int_{\partial K} \lambda_{\alpha}\left(S_{w, \alpha}^{*, h, n}\right) \boldsymbol{w}_{\alpha}^{h, n+1} \cdot \boldsymbol{n}=\int_{K} F_{\alpha}, \quad \alpha=w, n .
$$

4.2. Well-posedness of the decoupled problems in FC-IMPES-I scheme. For the FC-IMPES-I scheme, we can easily see the well-posedness of the problems in Step 1 and Step 3. In this section, we focus on the analysis for the well-posedness of the problem in Step 2 based on (3.12) and (3.13).

Given $\bar{S}_{w} \in Q_{h}$ and each phase velocity $\overline{\boldsymbol{u}}_{\alpha}, \alpha=w, n$, for any $\left(\boldsymbol{u}_{h}, p_{h}\right),\left(\boldsymbol{v}_{h}, q_{h}\right) \in \boldsymbol{U}_{h} \times Q_{h}$, we define the bilinear form

$$
b\left(\left(\boldsymbol{u}_{h}, p_{h}\right),\left(\boldsymbol{v}_{h}, q_{h}\right) ; \bar{S}_{w}\right)=a\left(\boldsymbol{u}_{h}, \boldsymbol{v}_{h}\right)-B\left(\boldsymbol{v}_{h}, q_{h}\right)-B_{t}\left(\boldsymbol{u}_{h}, q_{h} ; \bar{S}_{w}\right),
$$

where $a\left(\boldsymbol{u}_{h}, \boldsymbol{v}_{h}\right)=\left(\boldsymbol{K}^{-1} \boldsymbol{u}_{h}, \boldsymbol{v}_{h}\right), B\left(\boldsymbol{v}_{h}, q_{h}\right)=\left(\nabla \cdot \boldsymbol{v}_{h}, q_{h}\right)$ and the upwind values in $B_{\alpha}\left(\cdot, \cdot ; \bar{S}_{w}\right), \alpha=n, w$, are determined by the known phase velocity $\overline{\boldsymbol{u}}_{\alpha}$. Then the mixed finite element method based on (3.12) and (3.13) can be compactly rewritten as follows:

$$
b\left(\left(\boldsymbol{w}_{w}^{h, n+1}, p_{w}^{h, n+1}\right),(\boldsymbol{v}, q) ; S_{w}^{h, n}\right)=-\int_{\Gamma_{D}} p_{w}^{B} \boldsymbol{v} \cdot \boldsymbol{n}-\left(\rho_{w} g \nabla z, \boldsymbol{v}\right)-\left(F_{t}, q\right)+B_{n}\left(\boldsymbol{w}_{c}^{h, n+1}, q ; S_{w}^{h, n}\right) .
$$

For the velocity we will use the standard $L^{2}$-norm as $\|\cdot\|_{0}$, and for the pressure we will use the following norm:

$$
\left\|q_{h}\right\|_{1, h}^{2}=\sum_{F \in \mathcal{E}_{h}} h_{F}^{-1}\left\|\llbracket q_{h} \rrbracket\right\|_{0, F}^{2}, \quad q_{h} \in Q_{h} .
$$

The above problem in a compact form is well-posed based on the following full stability condition (cf. [6, 39] and [9, Section II.1]),

$$
\sup _{\left(\boldsymbol{v}_{h}, q_{h}\right) \in \boldsymbol{U}_{h} \times Q_{h}} \frac{b\left(\left(\boldsymbol{u}_{h}, p_{h}\right),\left(\boldsymbol{v}_{h}, q_{h}\right) ; \bar{S}_{w}\right)}{\left\|\boldsymbol{v}_{h}\right\|_{0}+\left\|q_{h}\right\|_{1, h}} \geq C_{s t a b}\left(\left\|\boldsymbol{u}_{h}\right\|_{0}+\left\|p_{h}\right\|_{1, h}\right), \quad\left(\boldsymbol{u}_{h}, p_{h}\right) \in \boldsymbol{U}_{h} \times Q_{h},
$$


where the constant $C_{\text {stab }}$ is a positive constant independent of mesh size. We define $\operatorname{Ker} B=\left\{\boldsymbol{v}_{h} \in \boldsymbol{U}_{h}\right.$ : $\left.B\left(\boldsymbol{v}_{h}, q_{h}\right)=0, q_{h} \in Q_{h}\right\}, \operatorname{Ker} B_{t}=\left\{\boldsymbol{v}_{h} \in \boldsymbol{U}_{h}: B_{t}\left(\boldsymbol{v}_{h}, q_{h} ; \bar{S}_{w}\right)=0, q_{h} \in Q_{h}\right\}$. It follows from [6,39] and [9, Section II.1] that the full stability condition is equivalent to the following invertibility condition

$$
\begin{aligned}
& \sup _{\boldsymbol{u}_{h} \in \operatorname{Ker} B} \frac{a\left(\boldsymbol{u}_{h}, \boldsymbol{v}_{h}\right)}{\left\|\boldsymbol{u}_{h}\right\|_{0}} \geq \alpha_{0}\left\|\boldsymbol{v}_{h}\right\|_{0}, \quad \boldsymbol{v}_{h} \in \operatorname{Ker} B_{t}, \\
& \sup _{\boldsymbol{v}_{h} \in \operatorname{Ker} B_{t}} \frac{a\left(\boldsymbol{u}_{h}, \boldsymbol{v}_{h}\right)}{\left\|\boldsymbol{v}_{h}\right\|_{0}} \geq \alpha_{1}\left\|\boldsymbol{u}_{h}\right\|_{0}, \quad \boldsymbol{u}_{h} \in \operatorname{Ker} B,
\end{aligned}
$$

and the Babuška-Brezzi stability conditions:

$$
\begin{aligned}
\sup _{\boldsymbol{v}_{h} \in \boldsymbol{U}_{h}} \frac{B\left(\boldsymbol{v}_{h}, q_{h}\right)}{\left\|\boldsymbol{v}_{h}\right\|_{0}} \geq C_{0}\left\|q_{h}\right\|_{1, h}, \quad q_{h} \in Q_{h}, \\
\sup _{\boldsymbol{v}_{h} \in \boldsymbol{U}_{h}} \frac{B_{t}\left(\boldsymbol{v}_{h}, q_{h} ; \bar{S}_{w}\right)}{\left\|\boldsymbol{v}_{h}\right\|_{0}} \geq C_{1}\left\|q_{h}\right\|_{1, h}, \quad q_{h} \in Q_{h} .
\end{aligned}
$$

Here the constants $\alpha_{0}, C_{0}, C_{1}$ are positive and independent of mesh size.

The stability estimate (4.4) has been proved in [39, Lemma 2.1]. Next we derive the estimates (4.2), (4.3) and (4.5).

Lemma 4.2. There exist positive constants $\alpha_{0}$ and $\alpha_{1}$ such that (4.2) and (4.3) hold.

Proof. For any $\boldsymbol{v}_{h} \in \operatorname{Ker} B_{t}$, we construct the following auxiliary problem: Find $\left(\boldsymbol{u}_{h}, p_{h}\right) \in \boldsymbol{U}_{h} \times Q_{h}$ such that

$$
\begin{aligned}
a\left(\boldsymbol{u}_{h}, \boldsymbol{w}_{h}\right)-B\left(\boldsymbol{w}_{h}, p_{h}\right) & =a\left(\boldsymbol{v}_{h}, \boldsymbol{w}_{h}\right), \quad \boldsymbol{w}_{h} \in \boldsymbol{U}_{h}, \\
B\left(\boldsymbol{u}_{h}, q_{h}\right) & =B_{t}\left(\boldsymbol{v}_{h}, q_{h} ; \bar{S}_{w}\right), \quad q_{h} \in Q_{h} .
\end{aligned}
$$

The well-posedness of the above saddle point problem is standard. By (4.7), we easily have $\boldsymbol{u}_{h} \in \operatorname{Ker} B$ and

$$
\left.\boldsymbol{u}_{h} \cdot \boldsymbol{n}\right|_{F}=\left.\left(\left.\lambda_{w}\left(\bar{S}_{w, w}^{*}\right)\right|_{F}+\left.\lambda_{n}\left(\bar{S}_{w, n}^{*}\right)\right|_{F}\right) \boldsymbol{v}_{h} \cdot \boldsymbol{n}\right|_{F}, \quad F \in \mathcal{E}_{h},
$$

where $\bar{S}_{w, w}^{*}$ is the upwind value of $\bar{S}_{w}$ according to the phase velocity $\overline{\boldsymbol{u}}_{w}$, and $\bar{S}_{w, n}^{*}=1-\bar{S}_{n}^{*}$ with $\bar{S}_{n}^{*}$ the upwind value of $\bar{S}_{n}=1-\bar{S}_{w}$ according to the phase velocity $\overline{\boldsymbol{u}}_{n}$.

For any $K \in \mathcal{T}_{h},\left.\boldsymbol{v}_{h}\right|_{K}=\left.\sum_{F \in \partial K}\left(\boldsymbol{v}_{h} \cdot \boldsymbol{n}\right)\right|_{F} \Phi_{F}$, where $\Phi_{F}$ is the lowest order Raviart-Thomas finite element basis function. By [18, Lemma A.1] and trace inequality, we have

$$
\left\|\boldsymbol{v}_{h}\right\|_{0, K}^{2} \leq \sum_{F \in \partial K}\left\|\left.\left(\boldsymbol{v}_{h} \cdot \boldsymbol{n}\right)\right|_{F} \Phi_{F}\right\|_{0, K}^{2} \leq C \sum_{F \in \partial K} h_{F}\left\|\boldsymbol{v}_{h} \cdot \boldsymbol{n}\right\|_{0, F}^{2} \leq C\left\|\boldsymbol{v}_{h}\right\|_{0, K}^{2}
$$

Thus, for any $K \in \mathcal{T}_{h}$, by the above estimates and (3.3) we further have

$$
\begin{aligned}
\left\|\boldsymbol{u}_{h}\right\|_{0, K}^{2} & \geq C \sum_{F \in \partial K} h_{F}\left\|\boldsymbol{u}_{h} \cdot \boldsymbol{n}\right\|_{0, F}^{2}=C \sum_{F \in \partial K} h_{F}\left\|\left(\lambda_{w}\left(\bar{S}_{w, w}^{*}\right)+\lambda_{n}\left(\bar{S}_{w, n}^{*}\right)\right) \boldsymbol{v}_{h} \cdot \boldsymbol{n}\right\|_{0, F}^{2} \\
& \geq C \sum_{F \in \partial K} h_{F}\left\|\boldsymbol{v}_{h} \cdot \boldsymbol{n}\right\|_{0, F}^{2} \geq C\left\|\boldsymbol{v}_{h}\right\|_{0, K}^{2} .
\end{aligned}
$$

Let $\boldsymbol{w}_{h}=\boldsymbol{u}_{h}$ in (4.6), we immediately have

$$
a\left(\boldsymbol{u}_{h}, \boldsymbol{v}_{h}\right)=a\left(\boldsymbol{u}_{h}, \boldsymbol{u}_{h}\right) \geq C\left\|\boldsymbol{u}_{h}\right\|_{0}^{2} \geq C\left\|\boldsymbol{u}_{h}\right\|_{0}\left\|\boldsymbol{v}_{h}\right\|_{0},
$$

which concludes the proof of (4.2). The estimate for (4.3) can be similarly derived, and we omit the details here.

Lemma 4.3. There exists a positive constant $C_{1}$ such that (4.5) holds.

Proof. By (4.4), for any $q_{h} \in Q_{h}$, there exists $\boldsymbol{w}_{h} \in \boldsymbol{U}_{h}$ such that

$$
B\left(\boldsymbol{w}_{h}, q_{h}\right) \geq C_{0}\left\|q_{h}\right\|_{1, h}\left\|\boldsymbol{w}_{h}\right\|_{0} .
$$

Let

$$
\left.\boldsymbol{w}_{h} \cdot \boldsymbol{n}\right|_{F}=\left.\left(\left.\lambda_{w}\left(\bar{S}_{w, w}^{*}\right)\right|_{F}+\left.\lambda_{n}\left(\bar{S}_{w, n}^{*}\right)\right|_{F}\right) \boldsymbol{v}_{h} \cdot \boldsymbol{n}\right|_{F}, \quad F \in \mathcal{E}_{h} .
$$


Clearly, we have

$$
B\left(\boldsymbol{w}_{h}, q_{h}\right)=B_{t}\left(\boldsymbol{v}_{h}, q_{h} ; \bar{S}_{w}\right) .
$$

Similar to the derivation in (4.8), we also have $\left\|\boldsymbol{w}_{h}\right\|_{0} \geq C\left\|\boldsymbol{v}_{h}\right\|_{0}$. Thus we finally have

$$
B_{t}\left(\boldsymbol{v}_{h}, q_{h} ; \bar{S}_{w}\right) \geq C_{1}\left\|q_{h}\right\|_{1, h}\left\|\boldsymbol{v}_{h}\right\|_{0},
$$

which concludes the proof.

4.3. Bounds-preserving property of saturation for both phases. In this section we state that the approximate saturation of each phase from the FC-IMPES-I scheme can be bounds-preserving if the time step size is smaller than a certain value. Since the local mass conservation for both phases holds, i.e., the approximate saturations of both phases satisfy the discrete mass-conservative law as in (3.4), the bounds of the wetting and non-wetting phase saturations can be similarly analyzed.

Now we propose two reasonable assumptions. Firstly, we assume that there exists a positive constant $\gamma_{\alpha}$ such that

$$
\lambda_{\alpha}\left(S_{\alpha}\right) \leq \gamma_{\alpha} S_{\alpha}, \quad \alpha=w, n .
$$

For instance, when we use the Brooks-Corey model [10] for the two-phase flow in porous media, the phase mobilities are nonlinear functions and defined as follows:

$$
\lambda_{w}\left(S_{w}\right)=\frac{1}{\mu_{w}} \bar{S}_{w}^{\frac{2+3 \theta}{\theta}}, \quad \lambda_{n}\left(S_{w}\right)=\frac{1}{\mu_{n}}\left(1-\bar{S}_{w}\right)^{2}\left(1-\bar{S}_{w}^{\frac{2+\theta}{\theta}}\right)
$$

where $\theta$ is the parameter associated with pore size distribution, $\bar{S}_{w}$ is the effective saturation defined as $\left(S_{w}-S_{r w}\right) /\left(1-S_{r n}-S_{r w}\right), S_{r w}$ and $S_{r n}$ are residual wetting phase saturation and residual non-wetting phase saturation. Let $\eta=\frac{2+3 \theta}{\theta}>1$, then we can easily derive that

$$
\lambda_{w}\left(S_{w}\right) \leq \frac{1}{\mu_{w}}\left(2 S_{w}\right)^{\eta} \leq \frac{2^{\eta}}{\mu_{w}} S_{w} .
$$

Thus we can choose $\gamma_{w}=\frac{2^{\eta}}{\mu_{w}}$ in (4.9). Similarly, we can also assume there exists a positive constant $\gamma_{n}$ such that $\lambda_{n}\left(S_{n}\right) \leq \gamma_{n} S_{n}$.

Next, if $F_{\alpha}$ in (2.1) is a sink term, i.e., $F_{\alpha} \leq 0$, we assume that there exist two positive constants $\beta_{1}$ and $\beta_{2}$ such that

$$
\beta_{1} S_{\alpha} \leq\left|F_{\alpha}\right| \leq \beta_{2} S_{\alpha}
$$

This assumption is reasonable since the sink term $F_{\alpha}$ comes from the mass flow rate.

Lemma 4.4. We assume the estimates (4.9) and (4.10) hold true. Let $\delta t=t_{n+1}-t_{n}$. For the approximate wetting phase saturation in the FC-IMPES-I scheme, we assume $S_{\alpha}^{h, n} \in(0,1)$ with tolerance saturation $S_{t \alpha}$, $\alpha=w, n$. For any $K \in \mathcal{T}_{h}$, if $\frac{\delta t}{h}$ is sufficiently small, we have

$$
S_{\alpha}^{h, n+1} \in(0,1), \quad \alpha=w, n .
$$

Proof. We focus on the analysis for the bounds of the wetting phase saturation. For any $K \in \mathcal{T}_{h}$, let $q=1$ in $\bar{K}$ and $q=0$ in $\Omega \backslash \bar{K}$ in (3.14), we immediately have

$$
\phi \frac{S_{w}^{h, n+1}-S_{w}^{h, n}}{\delta t}|K|=-\left(\int_{\widetilde{\partial K}_{w, n+1}^{-}}+\int_{\widetilde{\partial K}_{w, n+1}^{+}}\right) \lambda_{w}\left(S_{w, w}^{*, h, n}\right) \boldsymbol{w}_{w}^{h, n+1} \cdot \boldsymbol{n}+\bar{F}_{w}|K|, \quad \text { on } K,
$$

where $\widetilde{\partial K}_{w, n+1}^{-}=\left\{F \subset \partial K: \boldsymbol{w}_{w}^{h, n+1} \cdot \boldsymbol{n}<0\right\}, \widetilde{\partial K}_{w, n+1}^{+}=\left\{F \subset \partial K: \boldsymbol{w}_{w}^{h, n+1} \cdot \boldsymbol{n} \geq 0\right\}$ and $\bar{F}_{w}$ is the mean value of $F_{w}$ over $K$. By the shape regularity of the mesh, there holds

$$
\phi S_{w}^{h, n+1}=\phi S_{w}^{h, n}-\left(\sum_{F \in \widetilde{\partial K}_{w, n+1}^{+}}+\sum_{F \in \overline{\partial K}_{w, n+1}^{-}}\right) \frac{\delta t}{C_{F} h_{K}} \lambda_{w}\left(S_{w, w}^{*, h, n}\right) \boldsymbol{w}_{w}^{h, n+1} \cdot \boldsymbol{n}+\delta t \bar{F}_{w} \quad \text { on } K,
$$


where $C_{F}>0$ depends on the shape regularity of the mesh. If there is injection source or no source on $K$, we have $\bar{F}_{w} \geq 0$. Otherwise, $\bar{F}_{w}<0$ is production term, by the assumption in (4.10), we have $\bar{F}_{w} \geq-\beta_{2} S_{w}^{h, n+1}$. By the assumption (4.9), we derive that

$$
\begin{aligned}
& \sum_{F \in \widetilde{\partial K}_{w, n+1}^{+}} \frac{\delta t}{C_{F} h_{K}} \lambda_{w}\left(S_{w, w}^{*, h, n}\right) \boldsymbol{w}_{w}^{h, n+1} \cdot \boldsymbol{n}=\left(\sum_{F \in \widetilde{\partial K}_{w, n+1}^{+} \cap \partial K_{w, n}^{+}}+\sum_{F \in \widetilde{\partial K}_{w, n+1}^{+} \backslash \partial K_{w, n}^{+}}\right) \frac{\delta t}{C_{F} h_{K}} \lambda_{w}\left(S_{w, w}^{*, h, n}\right) \boldsymbol{w}_{w}^{h, n+1} \cdot \boldsymbol{n} \\
\leq & \sum_{F \in \widetilde{\partial K}_{w, n+1}^{+}} \frac{\delta t \gamma_{w}}{C_{F} h_{K}} S_{w}^{h, n} \boldsymbol{w}_{w}^{h, n+1} \cdot \boldsymbol{n}+\sum_{F \in \widetilde{\partial K}_{w, n+1}^{+} \backslash \partial K_{w, n}^{+}} \frac{\delta t \gamma_{w}}{C_{F} h_{K}}\left|S_{w}^{h, n}\right|_{K^{\prime}}-S_{w}^{h, n} \mid \boldsymbol{w}_{w}^{h, n+1} \cdot \boldsymbol{n},
\end{aligned}
$$

where $K^{\prime}$ is the neighboring element of $K$ and $\partial K_{w, n}^{+}$is the outflow edges of $\boldsymbol{w}_{w}^{h}$ on $\partial K$. For $F \in \widetilde{\partial K}_{w, n+1}^{+} \backslash$ $\partial K_{w, n}^{+}$, the phase velocity changes the direction on $F$ between the two time steps, then we can assume there exists a positive constant $\epsilon$ which is sufficiently small such that $\left|\boldsymbol{w}_{w}^{h, n+1} \cdot \boldsymbol{n}\right| \leq \epsilon$ on $F$. Combining the above estimates and noting the tolerance saturation assumption for $S_{w}^{h, n}$, we can observe that when $\frac{\delta t}{h}$ is small enough, we have $S_{w}^{h, n+1} \geq C S_{t w}>0$ on $K$.

By the similar approach, we also have $S_{n}^{h, n+1}>0$. Due to the fact that $S_{w}^{h, n+1}+S_{n}^{h, n+1}=1$, then we can easily combine this fact with the low bounds of $S_{n}^{h, n+1}$ and $S_{w}^{h, n+1}$ to obtain $S_{w}^{h, n+1}<1$ and $S_{n}^{h, n+1}<1$ and complete the proof.

\section{NumERICAL EXPERIMENTS}

Three numerical cases are designed in this paper to verify our scheme and to show the preference. For simplicity, we focus on the numerical verification of the FC-IMPES-I scheme, and the HF-IMPES scheme is tested in the following examples. As is popular in reservoir simulation, the random distribution of permeability has been taken into our account and we generate a permeability distribution based on matrix decomposition technique. We assume that the absolute permeability tensor is chosen as $\boldsymbol{K}=K \boldsymbol{I}$, where $\boldsymbol{I}$ is the identity matrix and $K$ is a positive real number. In the following experiments, the capillary pressure function is given by Hoteit and Firoozabadi in [27] as $p_{c}\left(S_{w}\right)=-\frac{B_{c}}{\sqrt{K}} \log \bar{S}_{w}$, where $B_{c}$ is a positive parameter with unit $1 \mathrm{bar} \cdot m d^{1 / 2}$ and $\bar{S}_{w}$ is the effective saturation defined as in Section 4.3 . We choose $B_{c}=25$ and assume the porosity of the porous media $\phi=0.2$ in all the following examples. Moreover, the relative permeabilities are given by $k_{r w}=\bar{S}_{w}^{\beta}$ and $k_{r n}=\left(1-\bar{S}_{w}\right)^{\beta}$, where $\beta$ is a positive integer number. In the experiments, we use the quadratic relative permeabilities, i.e., $\beta=2$.

The Buckely-Leverett problem in a 1D homogeneous porous medium with zero capillary pressure is firstly tested based on our algorithm. Then our algorithm is applied for a case in heterogeneous porous media to test the imbibition phenomena. The saturation profiles of wetting phase during the simulation process have been presented with reasonable results and the relative permeability of wetting and non-wetting phase have been illustrated to show the effectiveness of our scheme. A typical reservoir simulation process, SPE 10 case, has been modified and simulated using our scheme. The mass conservation property of our scheme is verified from two points of view: the injected wetting phase is equivalent to the current wetting phase saturation and the two approaches (cf. Step 3 in Algorithm 3.1) to calculate the saturation of non-wetting phase will result in the same value. Furthermore, a two phase counter flow case is designed and our scheme has shown much preferable results compared to the previous partially mass-conservative IMPES scheme. To demonstrate the conservation property of our algorithm, we set the time step as 1 day, which is preferable and applicable in industry.

Example 5.1. We firstly test the Buckely-Leverett problem in a 1D homogeneous porous medium with different fluid properties and zero capillary pressure (as presented in [27]). This problem has well-known analytical solution. We apply our algorithm to simulate this benchmark problem and compare the results with the case in [27]. We consider a 1D horizontal homogeneous domain of length $300 \mathrm{~m}$, initially saturated with oil (non-wetting phase). Water (wetting phase) is injected with a constant flow rate at one end to displace oil to the other end. The pressure is kept as a constant at the production end and the capillary pressure is neglected. The injection rate is $5 \times 10^{-4} \mathrm{PV} /$ day, and the rock porosity is 0.2 . Viscosity ratio 
between the two fluids is $2 / 3$, density ration is 1 and $k_{r w}=\bar{S}_{w}^{2}$, here we use the same data for Fig. 1.d in $[27]$.

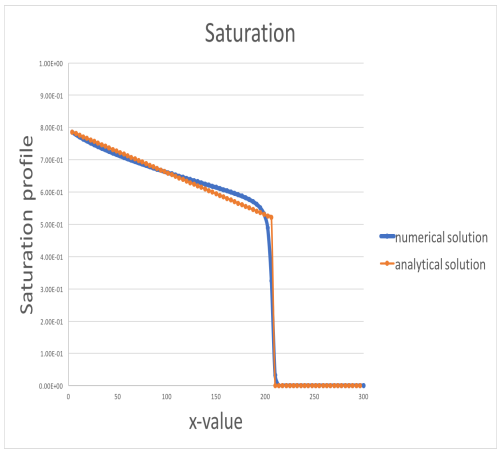

FiguRE 5.1. (Example 5.1) Wetting phase saturation curve.

\begin{tabular}{ccc}
\hline Time Step Size (day) & Relative Error & Order \\
\hline \hline 8 & $5.93 \times 10^{-2}$ & 1.12 \\
\hline 4 & $2.94 \times 10^{-2}$ & 1.01 \\
\hline 2 & $1.33 \times 10^{-2}$ & 1.15 \\
\hline 1 & $6.58 \times 10^{-3}$ & 1.02
\end{tabular}

TABLE 1. (Example 5.1) Convergence history with respect to the time step size on a fixed mesh $80 \times 1$.

\begin{tabular}{ccc}
\hline Spatial Mesh & Relative Error & Order \\
\hline \hline $80 \times 1$ & $6.58 \times 10^{-3}$ & 1.05 \\
\hline $40 \times 1$ & $1.36 \times 10^{-2}$ & 1.06 \\
\hline $20 \times 1$ & $2.83 \times 10^{-2}$ & 1.03 \\
\hline $10 \times 1$ & $5.76 \times 10^{-2}$ & 1.02 \\
\hline
\end{tabular}

TABLE 2. (Example 5.1) Convergence history with respect to the mesh size with a constant time step size at 1 day.
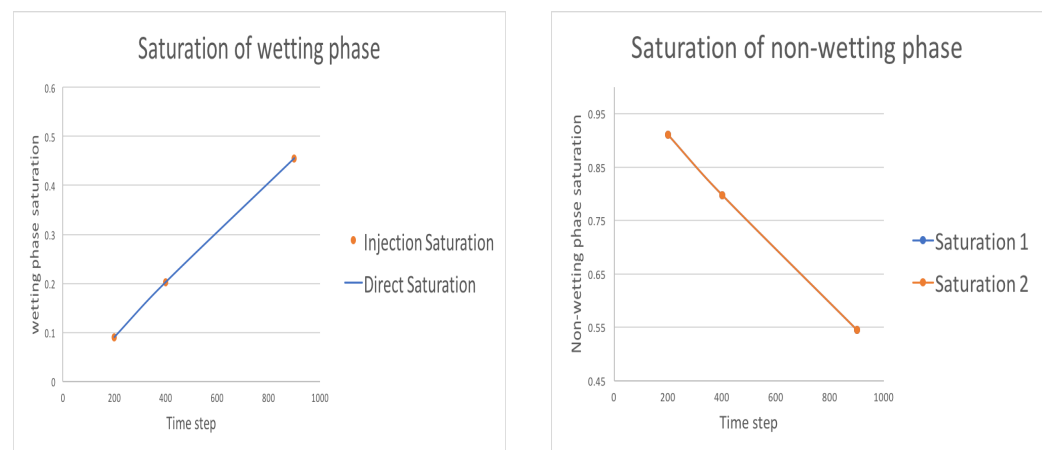

FIGURE 5.2. (Example 5.1) Left: wetting phase saturation. Right: non-wetting phase saturation.

After 900 days, the numerical and analytical solution curves of wetting phase saturation are presented in Figure 5.1. We observe that the numerical and analytical solutions meet well and the numerical solution 
also meets well with the result in Fig. 1.d of [27]. In order to test the convergence of the scheme, two kinds of convergence with respect to mesh size and time step size are designed. The first one is to test the convergence with respect to the time step size on a fixed mesh $80 \times 1$ and use four different time step sizes ( 1 day, 2 days, 4 days and 8 days). The second one is to test the convergence with respect to the mesh size with a fixed time step size 1 day and use four different spatial mesh sizes $(80 \times 1,40 \times 1,20 \times 1$ and $10 \times 1)$. The analytical solution of wetting phase saturation at 900 days are selected as the benchmark solution, and compared with all the cases. The relative errors of wetting phase saturation in $L^{2}$ norm are computed. From the convergence results shown in Table 1 and Table 2, it can be easily see that our scheme has a good convergence of first order with respect to the time step size and mesh size.

The mass conservation property of our algorithm can be verified from two points of view. As shown in the left graph of Figure 5.2, the modified wetting phase injection meets well with the wetting phase saturation in the process. The injection amount is modified via a similar concept of saturation calculation process, as divided by the total volume. In the right graph of Figure 5.2, the saturation of non-wetting phase is calculated using two approaches, as in (3.14-3.16), and the results meet very well. Both the two graphs in Figure 5.2 have verified the mass conservation property of our algorithm.

Example 5.2. In this example our algorithm is used to test a case with specific geometry setup, similar with the case 6.3 in [7]. As shown in Figure 5.3, the porous medium is set with heterogeneous permeability. The porous medium is initially saturated with non-wetting phase fluid. Wetting phase fluid is injected at the center of the right boundary with a constant rate $1.5 \mathrm{PV} /$ year and the outlet boundary is set at the left-top corner. The viscosity ration of wetting phase and non-wetting phase is set as $1 / 2$, and the density ration is 1 .

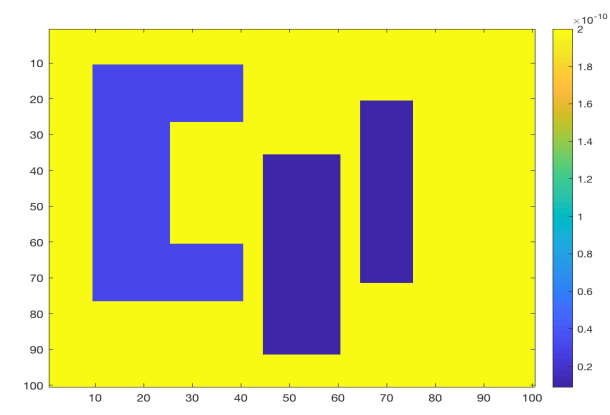

FiguRE 5.3. (Example 5.2) Permeability profile.
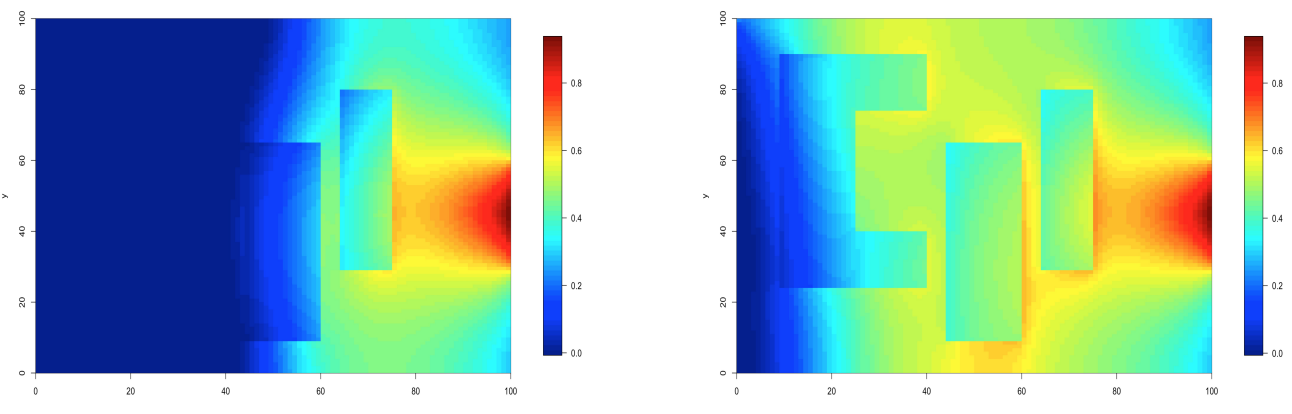

FiguRE 5.4. (Example 5.2) Saturation profile of wetting phase at 140 time step (left) and 920 time step (right).

To show the imbibition phenomena in the drainage process, the saturation profiles of wetting phase at time steps 140 and 320 are presented, as shown in Figure 5.4. The imbibition is obvious to find in the permeability gap area, and a clear cluster could be observed near the low permeability boundary. From the two graphs of Figure 5.3, we can see that the saturations calculated from the two different approaches as in (3.14-3.16) meet well, thus the mass conservation property of our algorithm is verified. 

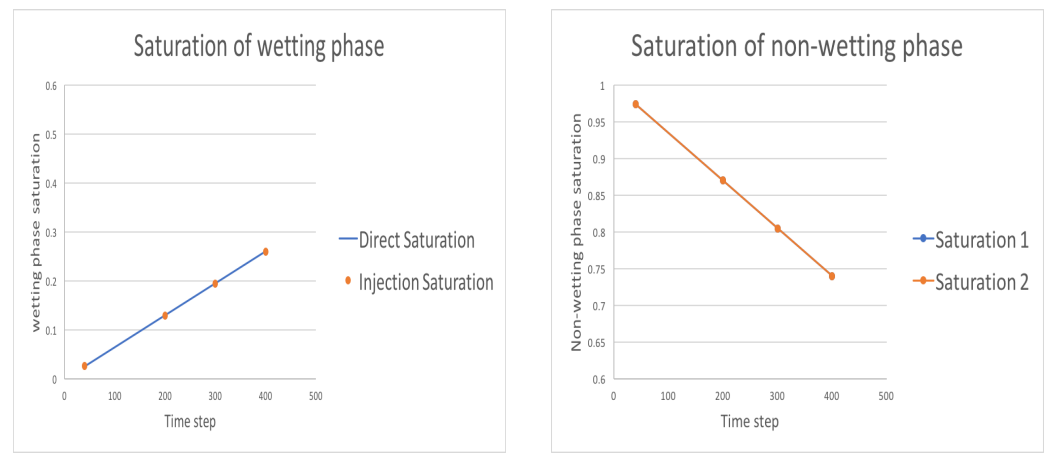

FigURE 5.5. (Example 5.2) Left: wetting phase saturation. Right: non-wetting phase saturation.

Example 5.3. In this example we simulate a drainage process of wetting phase with a generated permeability profile using a matrix decomposition technology. The density of wetting phase is set as $\rho_{W}=1000 \mathrm{~kg} / \mathrm{m}^{3}$ and the non-wetting phase is $\rho_{N}=660 \mathrm{~kg} / \mathrm{m}^{3}$. The viscosity of the wetting phase is set as $\mu_{W}=1 c P$ and the viscosity of the non-wetting phase is $\mu_{N}=0.45 \mathrm{cP}$. The injection is from the left-bottom point, with a rate of $0.1 \mathrm{PV} /$ year, as shown in the left graph of Figure 5.6. The simulation mesh is set as $100 \times 100$.

As discussed in [53], the probability statement of permeability $\boldsymbol{K}$ can be concerned as joint random variables, which could be defined as

$$
F_{K}\left(k_{1}, k_{2}, \ldots, k_{n}\right)=P\left(K_{1} \leq k_{1}, K_{2} \leq k_{2}, \ldots, K_{n} \leq k_{n}\right),
$$

where $P\left(K_{1} \leq k_{1}, K_{2} \leq k_{2}, \ldots, K_{n} \leq k_{n}\right)$ stands for the probability that the set of $n$ random variable $K_{i}$ takes a value less than the corresponding values of the set $k_{i}$. The multivariate probability density function $(\mathrm{PDF})$ is related to the cumulative (probability) distribution function $(\mathrm{CDF})$ as

$$
p_{K}\left(k_{1}, k_{2}, \ldots, k_{n}\right)=\frac{\partial^{n} F_{K}\left(k_{1}, k_{2}, \ldots, k_{n}\right)}{\partial k_{1} \partial k_{2} \cdots \partial k_{n}} .
$$

Permeability is generally viewed as subjected to the joint normal or lognormal random distribution. For normal distribution, the bivariate probability density function reads as

$$
p_{K_{1} K_{2}\left(k_{1}, k_{2}\right)}=\frac{1}{2 \pi \sigma_{1} \sigma_{2} \sqrt{1-\rho^{2}}} \exp \left(\frac{-1}{1-\rho^{2}}\left(\frac{\left(k_{1}-\mu_{1}\right)^{2}}{2 \sigma_{1}^{2}}-\rho \frac{\left(k_{1}-\mu_{1}\right)\left(k_{2}-\mu_{2}\right)}{\sigma_{1} \sigma_{2}}+\frac{\left(k_{2}-\mu_{2}\right)^{2}}{2 \sigma_{2}^{2}}\right)\right),
$$

where $\mu_{i}$ and $\sigma_{i}$ are the mean and standard variance of $k_{i}$, and $\rho$ is the correlation coefficient. In this paper, we assume that the permeability is subjected to a lognormal distribution, which could easily be referred from (5.1) as

$p_{K_{1} K_{2}\left(k_{1}, k_{2}\right)}=\frac{1}{2 \pi \sigma_{1} \sigma_{2} k_{1} k_{2} \sqrt{1-\rho^{2}}} \exp \left(\frac{-1}{1-\rho^{2}}\left(\frac{\left(\ln k_{1}-\mu_{1}\right)^{2}}{2 \sigma_{1}^{2}}-\rho \frac{\left(\ln k_{1}-\mu_{1}\right)\left(\ln k_{2}-\mu_{2}\right)}{\sigma_{1} \sigma_{2}}+\frac{\left(\ln k_{2}-\mu_{2}\right)^{2}}{2 \sigma_{2}^{2}}\right)\right)$.

As we have derived the PDF of permeability, the next step is to generate the random permeability value with known statistical properties, such as the mean, variance, and covariance. In particular, care must be taken in honoring the spatial structure of a random field as reflected in its covariance, since this quantity is difficult to reproduce compared to the mean and variance. How well the generated realizations honor the statistics of the random field of interest directly impacts the quality of the simulations. The realizations are said to be conditional if they honor available measurements in the field. There are several techniques available for generating random fields, including, but not limited to, the turning bands method, spectral methods, matrix decomposition, and Gaussian sequential simulation. Here, we use the matrix decomposition method.

Matrix decomposition is an elegant and direct method for generating correlated random fields in that the covariance matrix is used directly. For $n$ points on a grid where samples are to be generated, one can obtain the covariance matrix $\boldsymbol{C}$ of size $n \times n$ from the given covariance function $\boldsymbol{C}(r)$, where $r$ is the separation vector between two points. As discussed in [53], this covariance matrix $\boldsymbol{C}$ must be symmetric and positive definite. Hence, this matrix can be decomposed into a lower triangular matrix $\boldsymbol{L}$ and an upper triangular 
matrix $\boldsymbol{U}$ as follows:

$$
C=L U
$$

Since $\boldsymbol{C}$ is symmetric, we have $\boldsymbol{U}=\boldsymbol{L}^{T}$ in (5.2). A realization of the random field is obtained by multiplying the lower matrix $\boldsymbol{L}$ with a vector $\alpha=\left(\alpha_{1}, \ldots, \alpha_{n}\right)^{T}$ of $n$ independent, normally distributed random numbers with zero mean and unit variance,

$$
y=\boldsymbol{L} \alpha .
$$

The mean of the generated vector $y$ is zero and its covariance is $\left\langle y y^{T}\right\rangle=\boldsymbol{L}\left(\alpha \alpha^{\mathrm{T}}\right) \boldsymbol{U}=\boldsymbol{L I} \boldsymbol{U}=\boldsymbol{C}$. A nonzero (uniform or spatially varying) mean can be simply added to $y$ to obtain nonzero mean realizations. It is seen that once $\boldsymbol{C}$ is decomposed as in (5.2), a new realization $y$ is readily generated by (5.3) for a new vector $\alpha$, which can be created by any good random number generator. The additional cost to generate new realizations is small. Note that the method is independent of the dimensionality of the random field except for the size of the matrix. The generated permeability random distribution used in this paper is shown as the right of Figure 5.6. As the permeability value is relatively small, its logarithm is presented to make the illustration more clear.
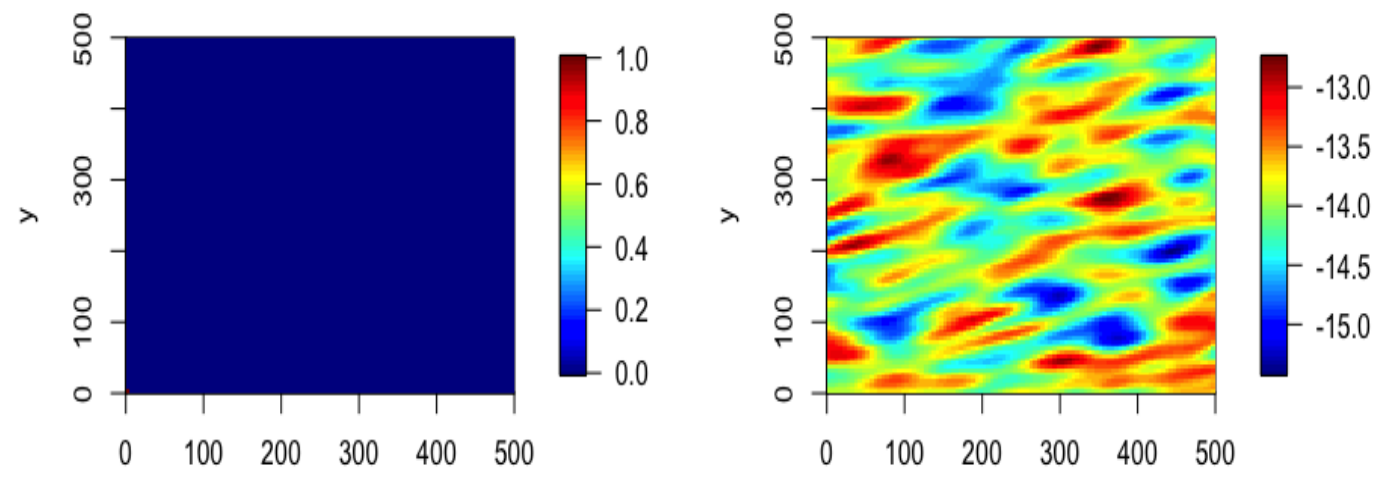

FIgURE 5.6. (Example 5.3) Left: wetting phase saturation at the initial station. Right: permeability.

To illustrate the drainage process, the saturation profile of wetting phase in the process is listed in Figure 5.7, at the time steps of 100,200, 500 and 1000, with flow quiver plotted as well. Generally speaking, the wetting phase flows from the left-bottom to the up-right, with different velocities at different directions due to the varieties of permeability. This process is meaningful and reasonable. We also plot the relative permeability of each phase with the change of averaged wetting phase saturation because the relative permeability curve is a classical way to check whether the solution is correct. As shown in left graph of Figure 5.8, we can see that a typical parabola is presented. From the right graph of Figure 5.8, we can see that the mass conservation property of our algorithm is also verified.

Example 5.4. We now apply our algorithm to simulate a modified version of SPE 10 data set which is obtained from the SPE website (http://www.spe.org/web/csp/). It could be concluded as a two phase flow (oil and gas) model that has a simple 2D vertical cross-sectional geometry with no dipping or faults. Due to the size limit of the original permeability file (a matrix of $65 \times 220 \times 80$ ), the mesh is set as $1 \times 100 \times 60$ with uniform size for each of the grid blocks. For the 2D plot, the $x$-axis is 22 meters long and 15.24 meters for $y$-axis. The density and viscosity of wetting and non-wetting phases are set as Example 5.3. The top of the model is at 0.0 meter with initial pressure at this point of $100 \mathrm{~Pa}$. Initially the model is fully saturated with oil (no connate water). The injection is also from the bottom-left point, with a rate of $6.97 \mathrm{~m}^{3} / \mathrm{day}$, as shown in the left graph of Figure 5.9. The permeability is shown in the right graph of Figure 5.9, and the logarithm value of permeability is presented to make the illustration more clear.

The drainage process at different time steps is illustrated in Figure 5.10, with flow quiver on it to show the main flow direction. Figure 5.11 shows the spatial average of wetting and non-wetting phase at different time steps. The mass conservation property of our algorithm can also be verified from two points of view. As shown in the two graphs of Figure 5.11, we can see that the saturations calculated using two approaches as 

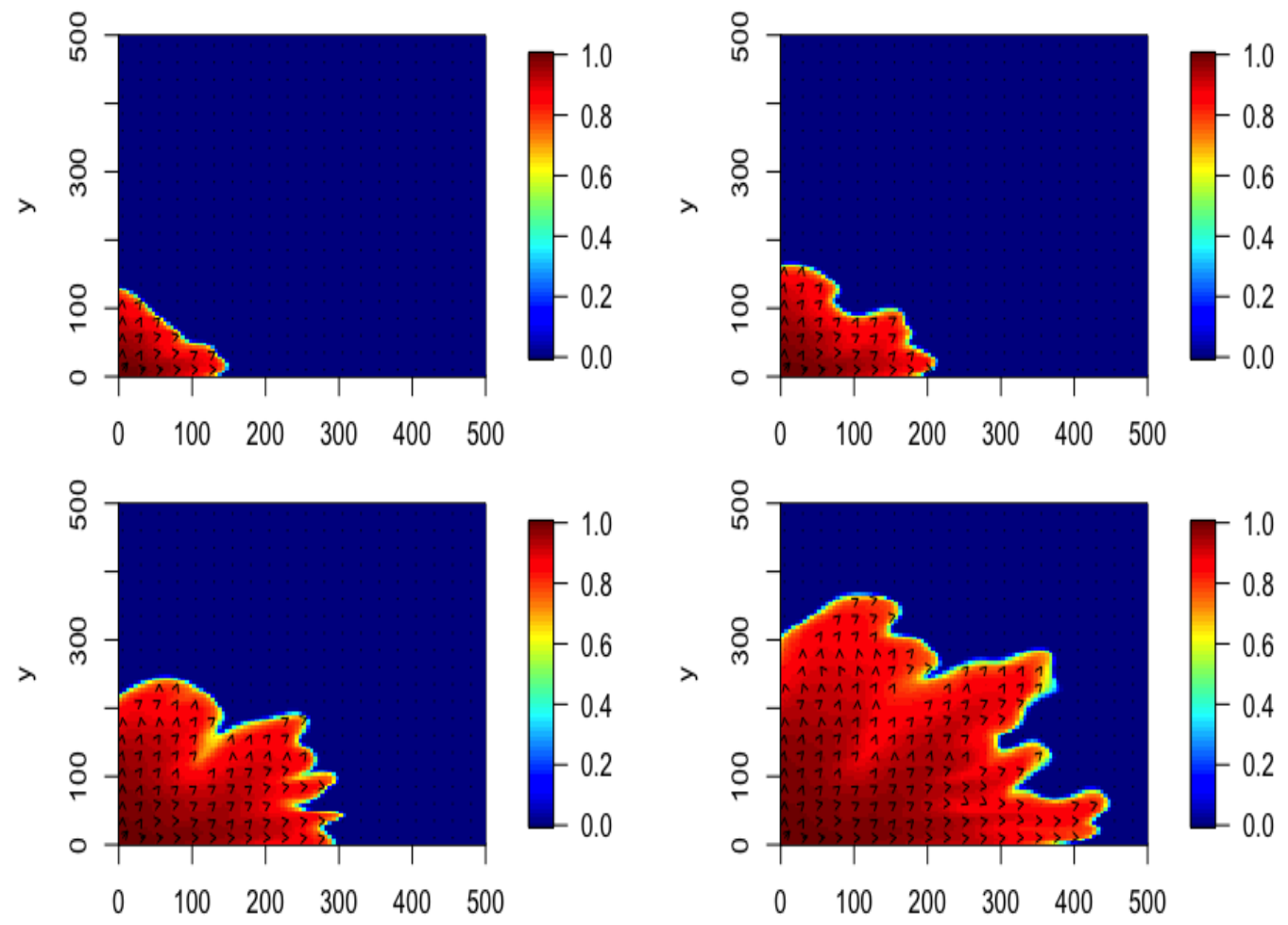

FIGURE 5.7. (Example 5.3) Saturation of wetting phase in the drainage process at the time steps 100, 200,500 and 1000 .
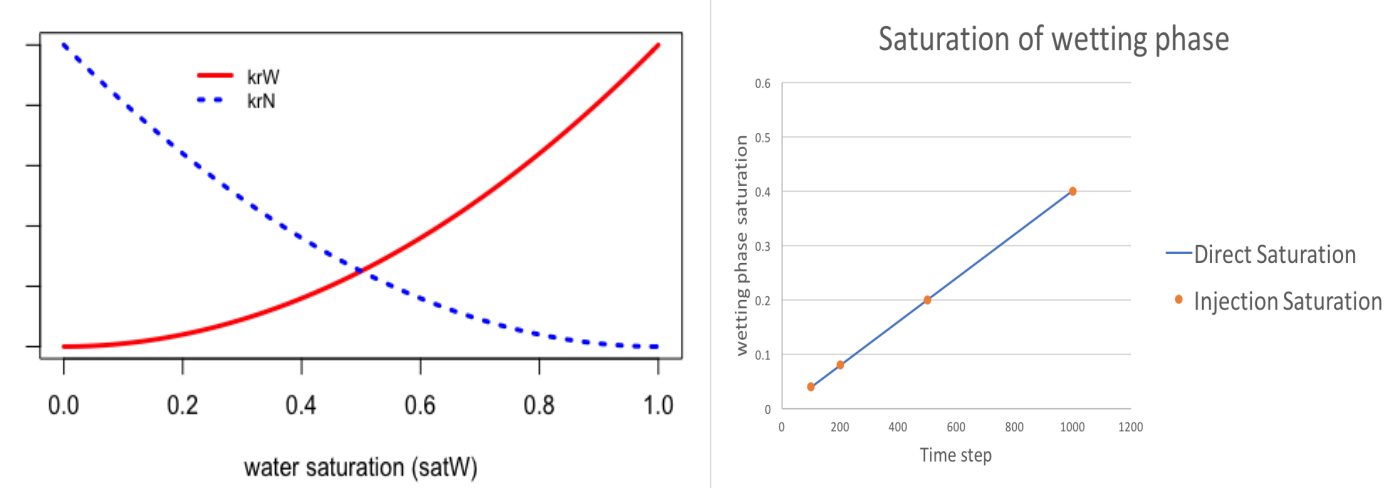

FIGURE 5.8. (Example 5.3) Left: plot of relative permeability via saturation of wetting and non-wetting phase. Right: wetting phase saturation.

in (3.14-3.16) meet very well. Thus both the two graphs in Figure 5.11 verify the mass conservation property of our algorithm.

Example 5.5. In this example our algorithm is expanded to a two-phase counter flow problem. As shown in Figure 5.12, the lower layer contains water, which is lighter than the heavy oil that fills out the rest of the domain, as shown in the left graph of Figure 5.8. The densities of water and heavy oil are $\rho_{W}=1000 \mathrm{~kg} / \mathrm{m}^{3}$ and $\rho_{N}=1050 \mathrm{~kg} / \mathrm{m}^{3}$ respectively, and the viscosities of water (wetting phase) and heavy oil (non-wetting phase) are set as $\mu_{W}=1 c P$ and $\mu_{N}=0.45 c P$. The gravity is taken into consideration in this case, varying with the above two cases. The permeability is also generated with the matrix decomposition technology introduced in Example 5.3 as a joint random distribution, as shown in the right graph of Figure 5.12. 

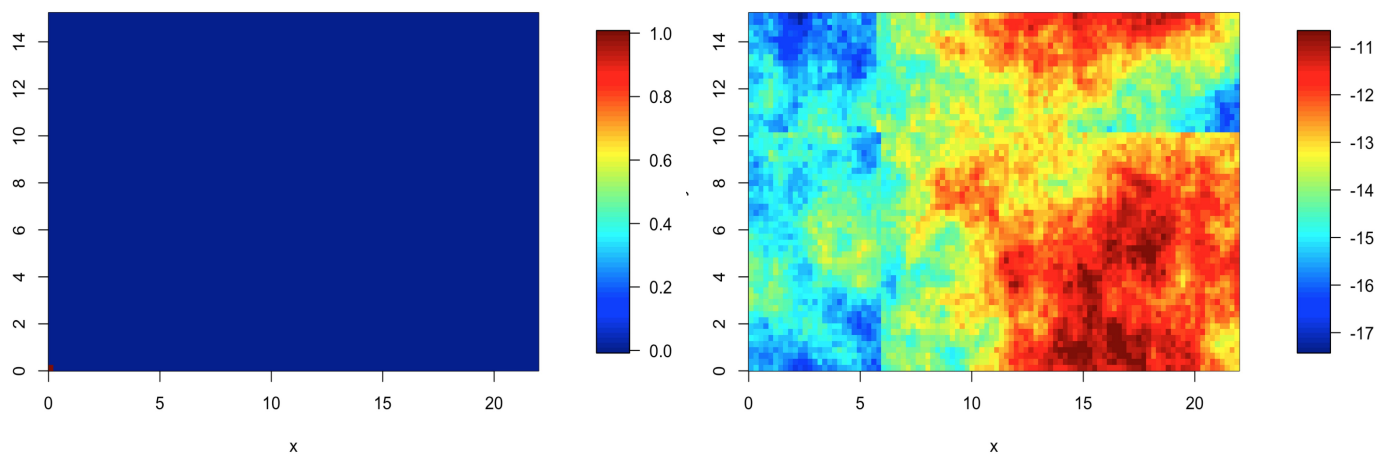

FIGURE 5.9. (Example 5.4) Left: wetting phase saturation at the initial station. Right: permeability.
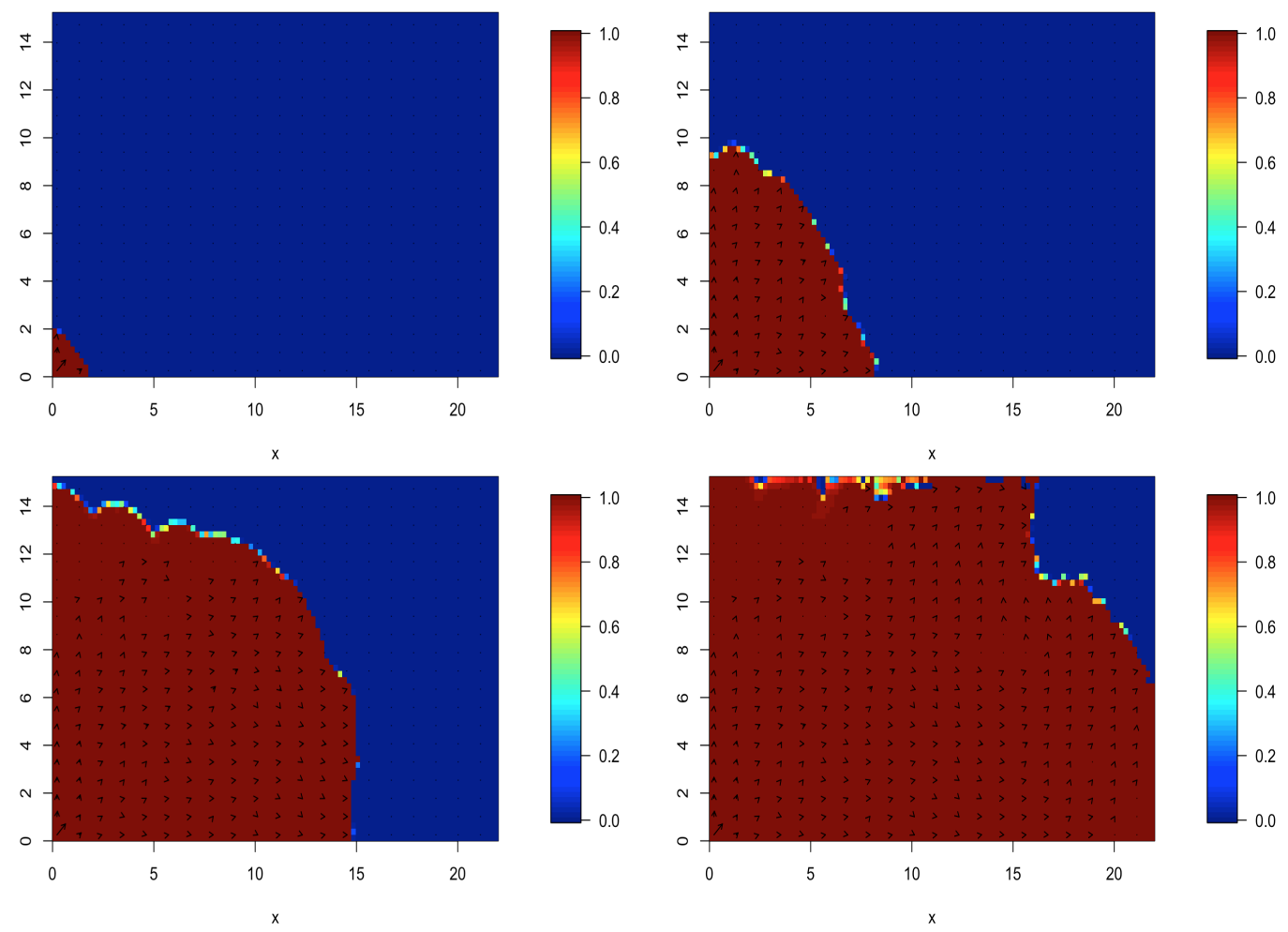

FigURE 5.10. (Example 5.4) Saturation of wetting phase in the drainage process at the time steps 10, 50,100 and 150 .

The drainage process is illustrated in Figure 5.13, while water (the wetting phase) raises under the effect of gravity, with different velocities on different direction due to the random distribution of permeability. The simulation with partially mass-conserved IMPES scheme is also conducted, as presented in Figure 5.14. It is easy to see that the results blow out, which means a bad non-physical result. As there is no injection or extraction in this case, the mean saturation of wetting phase should remain the same in the whole process, as presented in Figure 5.15. The conservation property of our scheme is verified again.

As the permeability distribution controls most of the flow process under the same initial and boundary conditions, another permeability distribution profile is tested to verify the robustness of our scheme, which is shown in Figure 5.16. A reasonable process with meaningful physical properties via our algorithm is presented in Figure 5.17, and the conservation of mean saturation is still kept well as shown in the left graph of Figure 5.18. Meanwhile, a non-physical process is also resulted from the partially mass-conservative IMPES scheme 

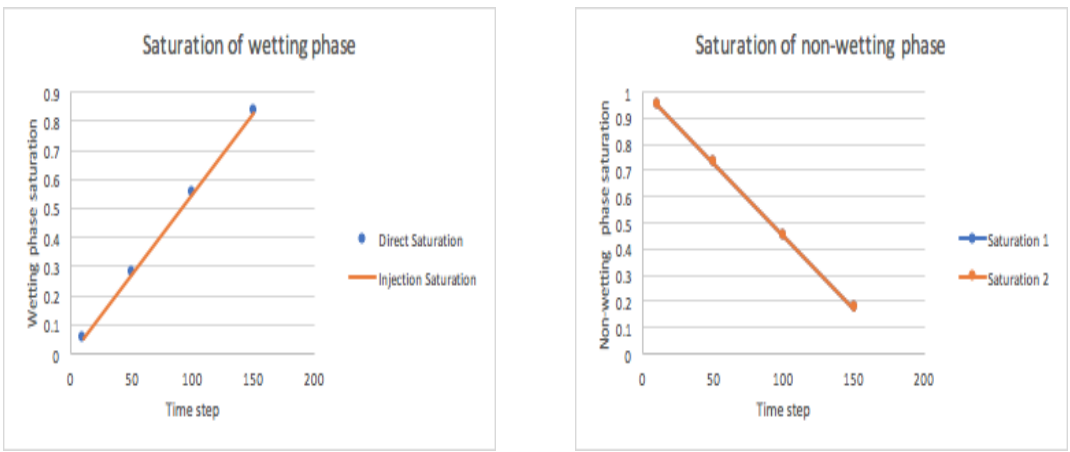

FIGURE 5.11. (Example 5.4) Left: wetting phase saturation. Right: non-wetting phase saturation.

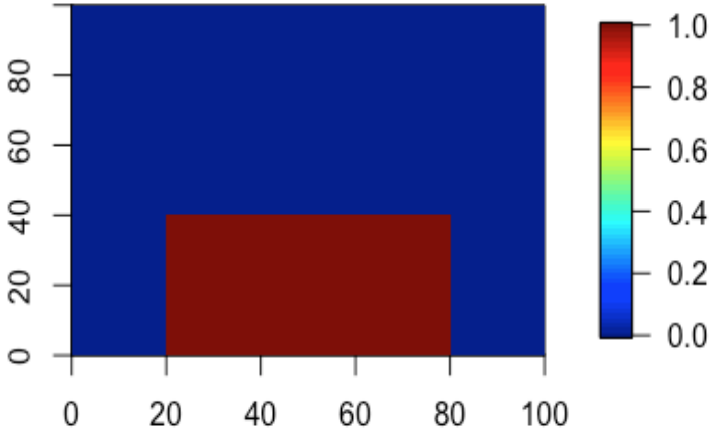

$\mathrm{X}$

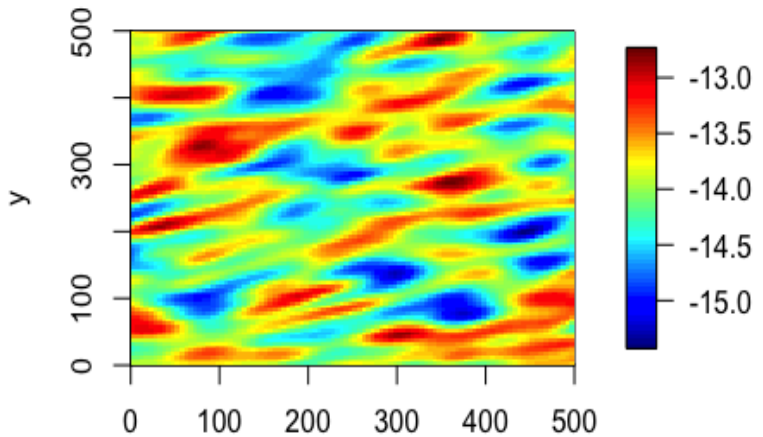

$\mathrm{X}$

FiguRE 5.12. (Example 5.5) Left: wetting phase saturation at the initial station. Right: permeability.
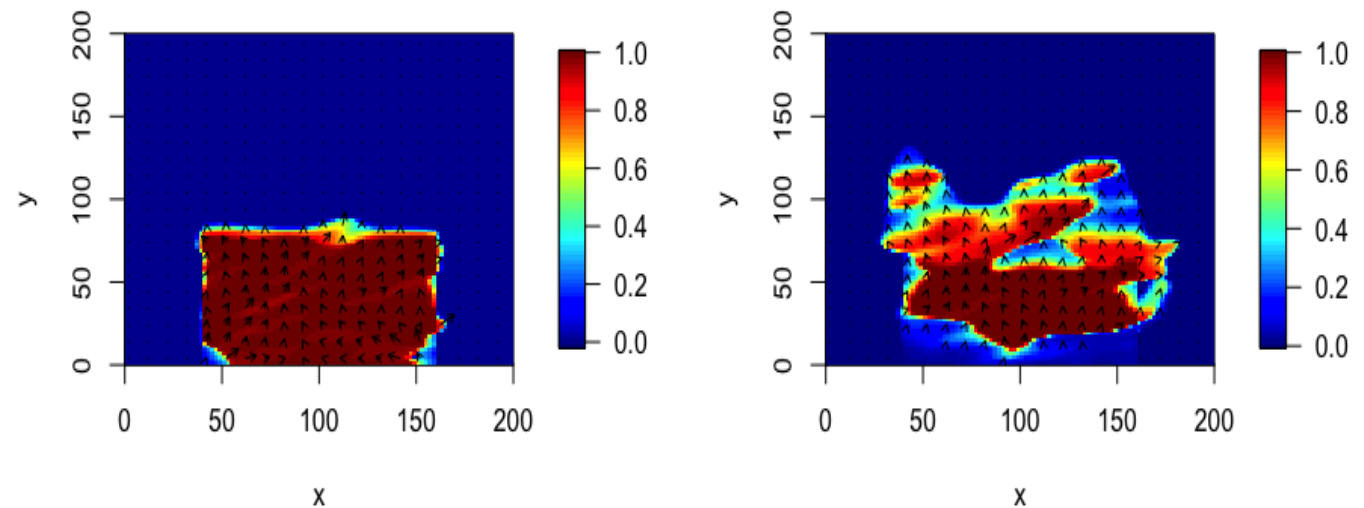

FigURE 5.13. (Example 5.5) Saturation of wetting phase in the drainage process at the time steps 10 and 300.

which can be observed from the right graph of Figure 5.18. Actually, the blow up in this graph is caused by the partially mass-conservative property of the HF-IMPES scheme, and thus the saturations computed by this classical IMPES scheme can not be bound-preserving. 

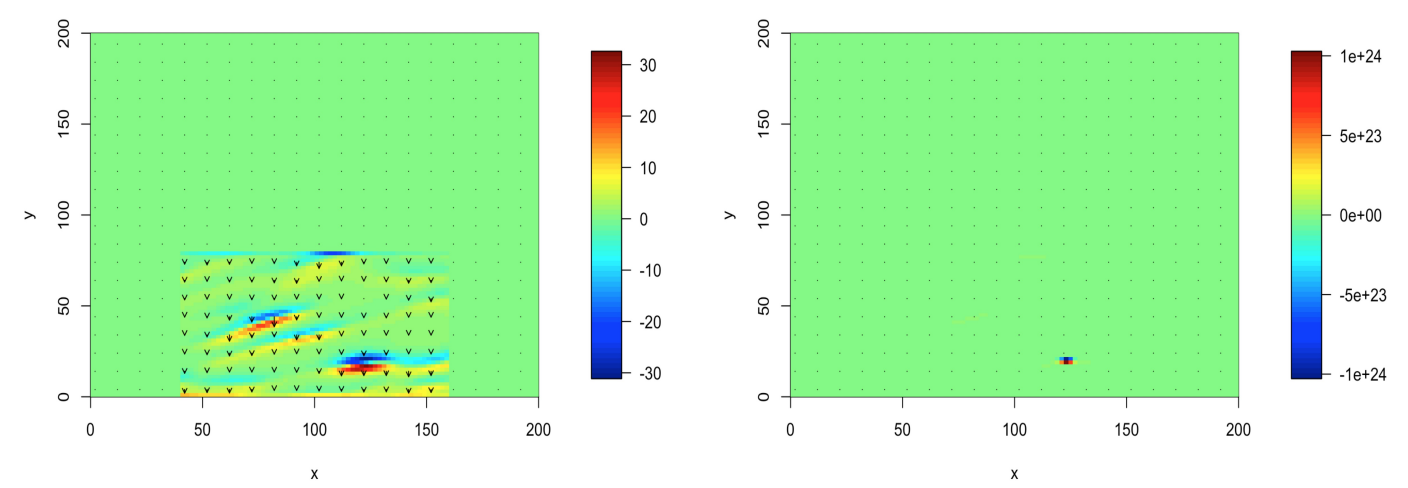

FIGURE 5.14. (Example 5.5) Wetting phase saturation using unconserved IMPES method at the time steps 10 and 20 .

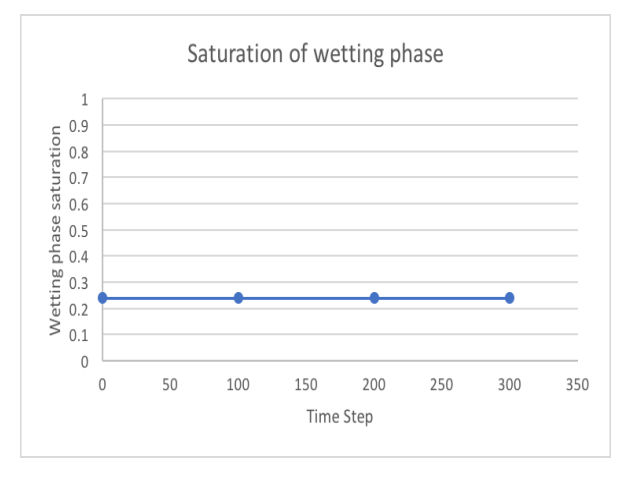

FiguRE 5.15. (Example 5.5) Mean saturation of wetting phase in drainage process.

Kxx

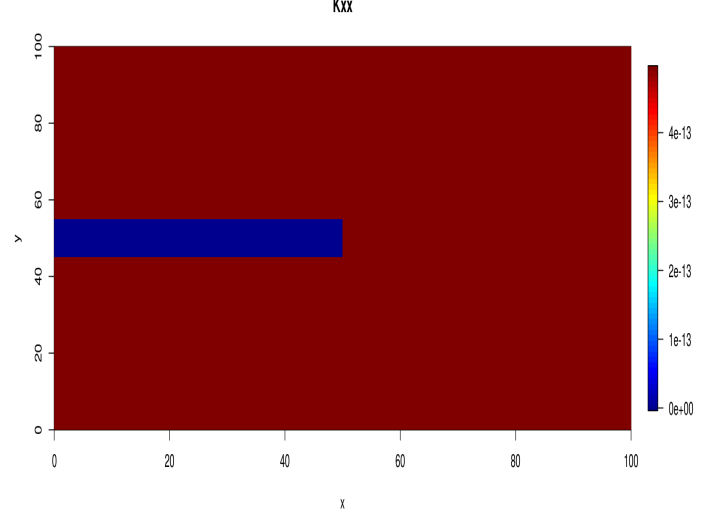

FIGURE 5.16. (Example 5.5) Another permeability distribution.

\section{Conclusion}

We aim to design fully mass-conservative IMPES schemes (FC-IMPES) for the simulation of incompressible and immiscible two-phase flow in porous media in this paper. Two kinds of FC-IMPES schemes are proposed and analyzed. The key idea is to sum the discretized conservation equation for each phase to obtain the total conservation equation, and a merit that the conservation of mass of both phases can be obtained. We also find that the total pressure-velocity system can be decoupled, and the problems in the decoupled 

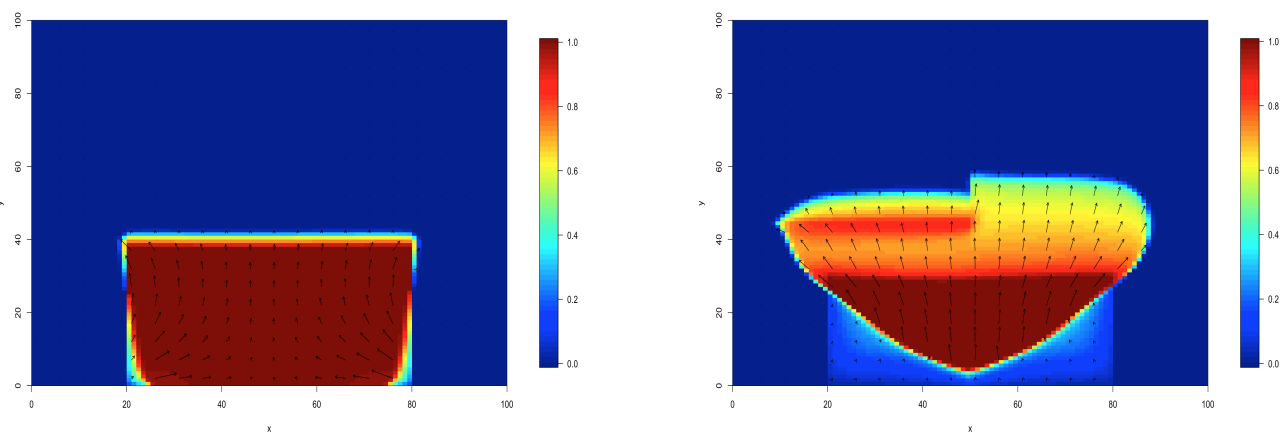

FiguRE 5.17. (Example 5.5) Saturation of wetting phase in the drainage process at the time steps 10 and 300.
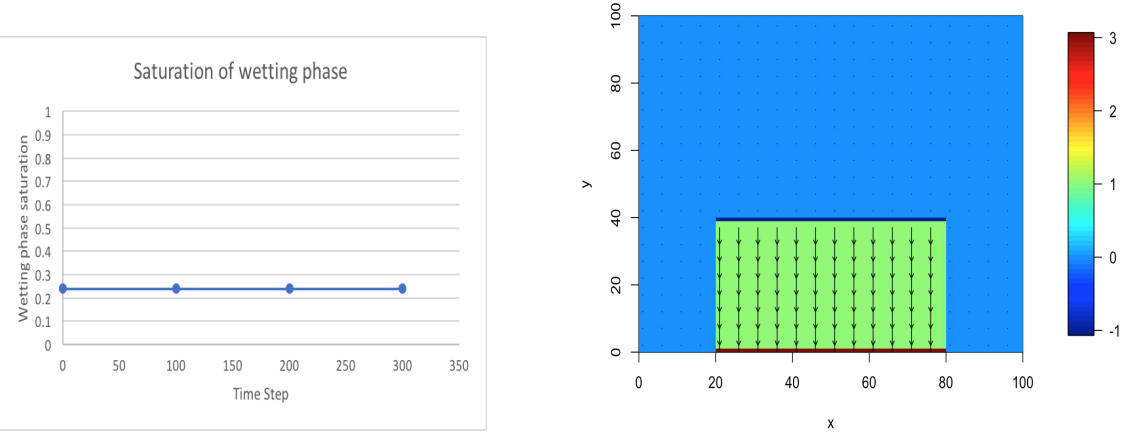

FIGURE 5.18. (Example 5.5) Left: plot of mean saturation via our algorithm. Right: results of partially mass-conservative scheme at the time steps 3 .

systems can be proved to be well-posed. Another merit of the new schemes is that the proposed FC-IMPES schemes are unbiased and the saturation of each phase can be proved to be bounds-preserving if the time step size is small enough. We test the FC-IMPES scheme of the first kind for three numerical examples and show that the new algorithms are more robust than the conventional IMPES schemes.

\section{REFERENCES}

[1] J. E. Aarnes, S. Krogstad, and K.-A. Lie, A hierarchical multiscale method for two-phase flow based upon mixed finite elements and nonuniform coarse grids, Multiscale Model. Simul., 5 (2006), pp. 337-363.

[2] R. Adams, Sobolev Spaces, Academic Press, New York, 1975.

[3] E. Abreu, J. Douglas, F. Furtado, and F. Pereira, Operator splitting for three-phase flow in heterogeneous porous media, Commun. Comput. Phys., 6 (2009), pp. 72-84.

[4] U. Ascher, S. J. Ruuth, and B. T. R. Wetton, Implicit-explicit methods for time-dependent partial differential equations, SIAM J. Numer. Anal., 32 (1995), pp. 797-823.

[5] K. Aziz and A. Settari, Petroleum Reservoir Simulation, London: Applied Science Pub., 1979.

[6] I. Babuška, J. Osborn, and J. Pitkäranta, Analysis of mixed methods using mesh dependent norms, Math. Comp., 35 (1980), pp. 1039-1062.

[7] P. Bastian and R. Helmig, Efficient fully-coupled solution techniques for two-phase flow in porous media: Parallel multigrid solution and large scale computations, Adv. Water Resour., 23 (1999), pp. 199-216.

[8] L. V. Branets, S. S. Ghai, S. L. Lyons, and X. Wu, Challenges and technologies in reservoir modeling, Commun. Comput. Phys., 6 (2009), pp. 1-23.

[9] F. Brezzi and M. Fortin, Mixed and Hybrid Finite Element Methods, Springer, NewYork, 1991.

[10] R. H. Brooks and T. Corey, Hydraulic Properties of Porous Media, in: Hydrol. Pap., vol. 3, 1964.

[11] Z. Chen, G. Huan, and B. Li, An improved IMPES method for two-phase flow in porous media, Transp. Porous Media, 54 (2004), pp. 361-376. 
[12] Z. Chen, G. Huan, and Y. Ma, Computational methods for multiphase flows in porous media, SIAM, Philadelphia, PA, USA, 2006.

[13] Z. Chen, Reservoir Simulation: Mathematical Techniques in Oil Recovery, SIAM, Philadelphia, PA, USA, 2007.

[14] K. H. Coats, A note on IMPES and some IMPES-based simulation models, Presented at the 15th symposium on reservoir simulation, Houston, TX, 1999, SPE 49774.

[15] K. H. Coats, IMPES stability: the CFL limit, Presented at the SPE reservoir simulation symposium, Houston, TX, 2001, SPE 85956.

[16] K. H. Coats, IMPES stability: selection of stable time steps, Presented at the SPE reservoir simulation symposium, Houston, TX, 2001, SPE 84924.

[17] D. A. Collins, L. X. Nghiem, Y. K. Li, and J. E. Grabenstetter, An efficient approach to adaptive implicit compositional simulation with an equation of state, SPE Reservoir Eng., 7 (1992), pp. 259-264.

[18] B. Cockburn and W. Zhang, An a posteriori error estimate for the variable-degree Raviart-Thomas method, Math. Comp., 83 (2014), pp. 1063-1082.

[19] C. N. Dawson, H. Klíe, M. F. Wheeler, and C. S. Woodward, A parallel, implicit, cell-centered method for two-phase flow with a preconditioned Newton-Krylov solver, Comput. Geosci., 1 (1997), pp. 215-249.

[20] L. J. Durlofsky, Y. Efendiev, and V. Ginting, An adaptive local-global multiscale finite volume element method for twophase flow simulations, Adv. Water Resour., 30 (2007), pp. 576-588.

[21] A. Ern, I. Mozolevski, and L. Schuh, Discontinuous Galerkin approximation of two-phase flows in heterogeneous porous media with discontinuous capillary pressures, Comput. Methods Appl. Mech. Engrg., 199 (2010), pp. 1491-1501.

[22] M. S. Fabien, M. G. Knepley, and B. M. Riviere, A hybridizable discontinuous Galerkin method for two-phase flow in heterogeneous porous media, arXiv preprint, arXiv:1802.06013, 2018.

[23] R. G. Fagin and Jr. C. H. Stewart, A new approach to the two-dimensional multiphase reservoir simulator, SPE J., 1966, SPE 1188.

[24] I. Faragó, A modified iterated operator splitting method, Appl. Math. Model., 32 (2008), pp. 1542-1551.

[25] J. Frank, W. Hundsdorfer, and J. G. Verwer, On the stability of implicit-explicit linear multistep methods, Appl. Numer. Math., 25 (1997), pp. 193-205.

[26] J. Geiser, Iterative operator-splitting methods with higher-order time integration methods and applications for parabolic partial differential equations, J. Comput. Appl. Math., 217 (2008), pp. 227-242.

[27] H. Hoteit and A. Firoozabadi, Numerical modeling of two-phase flow in heterogeneous permeable media with different capillarity pressures, Adv. Water Resour., 31 (2008), pp. 56-73.

[28] H. Hoteit and A. Firoozabadi, An efficient numerical model for incompressible two-phase flow in fractured media, Adv. Water Resour., 31 (2008), pp. 891-905.

[29] J. Hou, J. Chen, S. Sun, and Z. Chen, Adaptive mixed-hybrid and penalty discontinuous Galerkin method for two-phase flow in heterogeneous media, J. Comput. Appl. Math., 307 (2016), pp. 262-283.

[30] W. Hundsdorfer and S. J. Ruuth, IMEX extensions of linear multistep methods with general monotonicity and boundedness properties, J. Comput. Phys., 225 (2007), pp. 2016-2042.

[31] J. Kanney, C. Miller, and C. Kelley, Convergence of iterative split-operator approaches for approximating nonlinear reactive transport problems, Adv. Water Resources, 26 (2003), pp. 247-261.

[32] J. Kou and S. Sun, A new treatment of capillarity to improve the stability of IMPES two- phase flow formulation, Comput. Fluids, 39 (2010), pp. 1923-1931.

[33] J. Kou and S. Sun, On iterative IMPES formulation for two phase flow with capillarity in heterogeneous porous media, Internat. J. Numer. Anal. Model. Ser. B, 1 (2010), pp. 20-40.

[34] J. Kou and S. Sun, Convergence of discontinuous Galerkin methods for incompressible two-phase flow in heterogeneous media, SIAM J. Numer. Anal., 51 (2013), pp. 3280-3306.

[35] J. Kou and S. Sun, Upwind discontinuous Galerkin methods with conservation of mass of both phases for incompressible two-phase flow in porous media, Numer. Methods Partial Differ. Equ., 30 (2014), pp. 1674-1699.

[36] T. Koto, Stability of implicit-explicit linear multistep methods for ordinary and delay differential equations, Front. Math. China, 4 (2009), pp. 113-129.

[37] S. Lacroix, Y. V. Vassilevski, J. A. Wheeler, and M. F. Wheeler, Iterative solution methods for modeling multiphase flow in porous media fully implicitly, SIAM J. Sci. Comput., 25 (2003), pp. 905-926.

[38] D. Lanser and J. G. Verwer, Analysis of operator splitting for advection-diffusion-reaction problems from air pollution modelling, J. Comput. Appl. Math., 111 (1999), pp. 201-216.

[39] C. Lovadina and R. Stenberg, Energy norm a posteriori error estimates for mixed finite element methods, Math. Comp., 75 (2006), pp. 1659-1674.

[40] B. Lu, Iteratively Coupled Reservoir Simulation for Multiphase Flow in Porous Media, PhD dissertation, The University of Texas at Austin, 2008.

[41] B. Lu and M. F. Wheeler, Iterative coupling reservoir simulation on high performance computers, Pet. Sci., 6 (2009), pp. 43-50.

[42] Q. Lu, A Parallel Multiblock/Multiphysics Approach for Multiphase Flow in Porous Media, Ph.D Thesis, The University of Texas at Austin, 2000.

[43] J. E. P. Monteagudo and A. Firoozabadi, Comparison of fully implicit and IMPES formulations for simulation of water injection in fractured and unfractured media, Int. J. Numer. Meth. Eng., 69 (2007), pp. 698-728.

[44] M. Presho and J. Galvis, A mass conservative Generalized Multiscale Finite Element Method applied to two-phase flow in heterogeneous porous media, J. Comput. Appl. Math., 296 (2016), pp. 376-388. 
[45] J. W. Sheldon, B. Zondek, and W. T. Cardwell, One-dimensional, incompressible, noncapillary, two-phase fluid flow in a porous medium, T. SPE AIME, 216 (1959), pp. 290-296.

[46] H. L. Stone and A. O. Garder Jr., Analysis of gas-cap or dissolved-gas reservoirs, T. SPE AIME, 222 (1961), pp. 92-104.

[47] J. W. Watts, A compositional formulation of the pressure and saturation equations, 1985, SPE 12244.

[48] Y. Wu and G. Qin, A generalized numerical approach for modeling multiphase flow and transport in fractured porous media, Commun. Comput. Phys., 6 (2009), pp. 85-108.

[49] H. Yang, S. Sun, C. Yang, Nonlinearly preconditioned semismooth Newton methods for variational inequality solution of two-phase flow in porous media, J. Comput. Phys., 332 (2017), 1-20.

[50] H. Yang, C. Yang, S. Sun, Active-set reduced-space methods with nonlinear elimination for two-phase flow problems in porous media, SIAM J. Sci. Comput., 38 (2016), B593-B618.

[51] H. Yang, S. Sun, Y. Li, and C. Yang, A scalable fully implicit framework for reservoir simulation on parallel computers, Comput. Methods Appl. Mech. Engrg., 330 (2018), pp. 334-350.

[52] L. C. Young and R. E. Stephenson, A generalized compositional approach for reservoir simulation, SPE J, 23 (1983), pp. $727-742$.

[53] D. Zhang, Stochastic Methods for Flow in Porous Media: Coping with Uncertainties, Elsevier, 2001.

[54] A. Zidane and A. Firoozabadi, An implicit numerical model for multicomponent compressible two-phase flow in porous media, Adv. Water Resour., 85 (2015), pp. 64-78.

School of Mathematical Sciences and Fujian Provincial Key Laboratory on Mathematical Modeling and High Performance Scientific Computing, Xiamen University, Fujian, 361005, China

E-mail address: chx@xmu.edu.cn

School of Mathematics and Statistics, Hubei Engineering University, Xiaogan 432000, Hubei, China

E-mail address: jkou@hbeu.edu.cn

Computational Transport Phenomena Laboratory, Division of Physical Science and Engineering, King Abdullah

University of Science and Technology, Thuwal 23955-6900, Kingdom of Saudi Arabia

E-mail address: shuyu.sun@kaust.edu.sa

Computational Transport Phenomena Laboratory, Division of Physical Science and Engineering, King Abdullah University of Science and Technology, Thuwal 23955-6900, Kingdom of Saudi Arabia

E-mail address: tao.zhang.1@kaust.edu.sa 